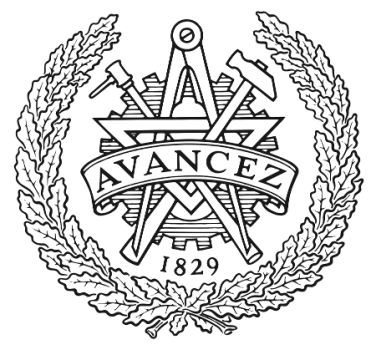

CHALMERS

UNIVERSITY OF TECHNOLOGY

\title{
Harnessing NLOS Components for Position and Orientation Estimation in 5G Millimeter Wave MIMO
}

Downloaded from: https://research.chalmers.se, 2023-04-26 10:48 UTC

Citation for the original published paper (version of record):

Mendrzik, R., Wymeersch, H., Bauch, G. et al (2019). Harnessing NLOS Components for Position and Orientation Estimation in 5G Millimeter Wave MIMO. IEEE Transactions on Wireless Communications, 18(1): 93-107. http://dx.doi.org/10.1109/TWC.2018.2877615

N.B. When citing this work, cite the original published paper.

(O2019 IEEE. Personal use of this material is permitted.

However, permission to reprint/republish this material for advertising or promotional purposes 


\title{
Harnessing NLOS Components for Position and Orientation Estimation in 5G Millimeter Wave MIMO
}

\author{
Rico Mendrzik, Student Member, IEEE, Henk Wymeersch, Member, IEEE, Gerhard Bauch, Fellow, IEEE, \\ Zohair Abu-Shaban, Member, IEEE
}

\begin{abstract}
In the past, NLOS propagation was proven to be a source of distortion for radio-based positioning systems due to the lack of temporal and spatial resolution of previous cellular systems. Hence every NLOS component was perceived as a perturbation for localization. Even though $5 \mathrm{G}$ is not yet standardized, a strong proposal, which has the potential to overcome the problem of limited temporal and spatial resolution, is the massive MIMO millimeter wave technology. We reconsider the role of NLOS components for position and orientation estimation in 5G millimeter wave MIMO systems. Our analysis is based on the concept of Fisher information. We show that, for sufficiently high temporal and spatial resolution, NLOS components always provide position and orientation information which consequently increases the position and orientation estimation accuracy. Additionally, we show that the information gain of NLOS components depends on the actual location of the reflector or scatter. Our numerical examples suggest that NLOS components are most informative about the position and orientation of a mobile terminal when the corresponding reflectors or scatterers are illuminated with narrow beams.
\end{abstract}

Index Terms-5G, millimeter wave communication, MIMO, position error bound, Fisher information

\section{INTRODUCTION}

\section{A. Motivation and State of the Art}

I $\mathrm{N}$ many conventional wireless networks, multipath (MP) propagation is considered as a distorting effect, which cannot be leveraged for positioning of network nodes, when no prior information regarding the location of the corresponding point of incidence ${ }^{1}$ is available [1]-[5]. The reason is that the information enclosed in the waveform of the received signal is not rich enough to resolve the non-line-of-sight (NLOS) components in space and time. The fifth generation $(5 \mathrm{G})$ networks are expected to use signals in the millimeter wave (mmWave) band [6] and employ massive multiple input multiple output (MIMO) to compensate for the high path loss [7], [8]. Particularly, mmWave MIMO systems operate at a

R. Mendrzik and G. Bauch are with the Institute of Communications, Hamburg University of Technology, Hamburg, 21073 Germany. H. Wymeersch is with the Department of Electrical Engineering, Chalmers University, Gothenburg, Sweden. Zohair Abu-Shaban is with the School of Engineering and Information Technology at the University of New South Wales, Canberra, Australia. This work is supported, in part, by the Horizon2020 projects 5GCAR and HIGHTS (MG-3.5a-2014-636537), and the VINNOVA COPPLAR project, funded under Strategic Vehicle Research and Innovation Grant No. 2015-04849.

${ }^{1}$ In order to cover both reflectors and scatterers, we use the term point of incidence in place of the location of a scatterer and the point of reflection of a reflector. carrier frequency beyond $28 \mathrm{GHz}$ using a large number of antennas at the base station and the mobile terminal [6], [9][12]. In the mmWave band, large contiguous frequency blocks are available which enable the support of high data rates [8], [13]. The large bandwidth in the mmWave band [12] results in high temporal resolution [14]. Moreover, the short wavelength of mmWave signals makes it possible to accommodate a large number of antennas in a small area [9], [15]. Hence large antenna arrays can be expected for base stations as well as mobile terminals. Large antenna arrays, in turn, allow for extremely narrow beams which enable accurate spatial resolution in the angular domain [16], [17]. Even though the positioning capabilities of mmWave MIMO in 5G are not yet fully explored, the high temporal and spatial resolutions of mmWave MIMO systems suggest that NLOS components can be resolved and harnessed for position and orientation estimation.

The fundamental limits of position and orientation estimation using mmWave MIMO in $5 \mathrm{G}$ have been recently investigated in [18]-[20]. In [18], a single anchor localization scheme was presented for indoor scenarios. The Fisher information matrix (FIM) of the position and orientation parameters as well as the NLOS parameters was presented. Based on this FIM, the position error bound (PEB) and orientation error bound (OEB) were derived numerically for different array configurations. It was shown in [18] that increasing the number of antenna array elements increases the localization accuracy. In [19], the FIM of all channel parameters was presented. Using the geometric relationship of the channel parameters and the position and orientation-related parameters, the FIM of the position and orientation-related parameters was derived in closed-form. Moreover, the PEB and the OEB were determined numerically, and algorithms which attain the previously determined bounds were also presented. It was shown numerically that even in the absence of the line-of-sight (LOS) path, positioning with reasonable accuracy is possible. In [20], fundamental limits of position and orientation estimation for uplink and downlink in 3D-space were presented. The FIM of the channel parameters was derived in a closed form similar to [19], which provided the FIM of the 2D channel parameters. Moreover, the structure of this FIM was analyzed and it was shown to become block diagonal when the bandwidth and the number of receive and transmit antennas are sufficiently large. In contrast to [19], which considered uniform linear arrays, [20] presented the derivation of the PEB and the OEB in closed-form for 
arbitrary antenna array structure. The PEB and the OEB were derived in a similar manner compared to [19]. In addition, the influence of different array types on the PEB and the OEB was investigated. Moreover, differences in the uplink and downlink were also considered in [20].

NLOS components have already been proven to be useful for indoor navigation [21]-[25]. In [21], [22], a two-stage approach is adopted to estimate and track the position of a mobile terminal and the positions of the so-called virtual anchors. Virtual anchors mimic an LOS transmission for every NLOS component. In the first stage of the approach in [21], [22], the complex channel gains and delays are determined. Based on these results, the position of the mobile terminal and the locations of the virtual anchors are estimated and tracked. The approaches in [23]-[25] leverage the huge bandwidth of ultra-wideband (UWB) signals in order to resolve NLOS components in time. NLOS components can be associated with virtual anchors using, e.g., belief propagation [23] or optimal sub-pattern assignment [24]. In order to reliably associate NLOS components with virtual anchors, multiple observations and mobility of the receiver are required. Virtual anchors and the unknown position of the mobile terminal are tracked over time using different filters such as, e.g., belief propagation [23], [26] or the extended Kalman filter [24]. The key difference of mmWave MIMO schemes in comparison to the works in [21]-[25] is that the former methods do not rely on the mobility of the mobile terminal to harness information from NLOS components. A snapshot (one transmission burst from the base station) is sufficient to exploit the information which NLOS components provide.

\section{B. Contribution and Paper Organization}

In [18]-[20], it was numerically shown that position and orientation estimation accuracy can benefit from NLOS components. However, the influence of the location of the base station, mobile terminal, and points of incidence of NLOS components is not well understood. The convoluted structure of the FIM of the channel parameters makes the analysis of the impact of NLOS components complicated. In our work, we build upon [20] and employ a simplified FIM of the channel parameters. Using a geometric transformation like in [19], [20], we obtain a simplified FIM in the position, orientation, and points of incidence domain. In order to study the impact of NLOS components on the position and orientation estimation accuracy, we use the notion of the equivalent FIM (EFIM) [27]. Firstly, we determine the EFIM of the position and orientation. Then, we decompose this EFIM in order to analyze and reveal the impact of NLOS components. Our contributions are summarized as follows:

- Assuming a large number of receive and transmit antennas as well as a large bandwidth, we derive an expression for the EFIM of the position and orientation, and we show that this EFIM can be written as the sum of rank one matrices, where each NLOS component contributes a distinct rank one matrix to the EFIM. ${ }^{2}$

- We show that this contribution from each NLOS component increases the position and orientation information content in the EFIM, and thus reduces the PEB and OEB. We show that NLOS components provide significant position and orientation information if and only if angleof-arrival (AOA), angle-of-departure (AOD), and time-ofarrival (TOA) can be estimated accurately.

- We derive the amount and direction of information ${ }^{3}$ in a closed form showing its relation to the geometry.

The rest of the paper is organized as follows. Section II discusses our system model, and Section III reviews the simplified FIM of the channel parameters from [20]. Our main results are presented in Section IV, where we derive the EFIM of the position and orientation, decompose the EFIM, and present the information gain from NLOS components. Section $\mathrm{V}$ contains numerical examples. The paper is concluded in Section VI.

Notation: Throughout this paper, we will stick to the following notational conventions. Scalars are denoted in italic, e.g., $x$. Lower case boldface indicates a column vector, e.g., $\mathbf{x}$, while upper case boldface denotes a matrix, e.g., X. Matrix elements are denoted by $[\mathbf{X}]_{i, j}$ where $i$ refers to rows and $j$ refers to columns, while $[\mathbf{X}]_{i: l, j: k}$ selects the sub-matrix of $\mathbf{X}$ between the rows $i$ to $l$ and the columns $j$ to $k$. Matrix transpose is indicated by superscript $\mathrm{T}$, e.g., $\mathbf{X}^{\mathrm{T}}$, while the superscript $\mathrm{H}$ refers to the transpose conjugate complex. Matrix trace is expressed by $\operatorname{tr}(\mathbf{X})$ and matrix determinant is indicated as $|\mathbf{X}|$. The Euclidean norm is denoted by $\|\cdot\|$, e.g., $\|\mathbf{x}\|$.

\section{SySTEM MODEL}

In this section, we first describe the geometry of the considered problem. Secondly, we specify the transmitter and the channel models. We conclude the section with the model of the receiver and a discussion on system-level aspects.

\section{A. Geometry}

We consider a mobile terminal which aims to estimate its own location and orientation in 2D space, based on the downlink signal received from the base station. The position and orientation of the base station are perfectly known to the mobile terminal. This information can be piggybacked in the header of a package without increasing the overhead notably. Even though our analysis is focused on downlink position and orientation estimation, the estimation could also be carried out in the uplink as presented in [20]. We assume that mobile terminal and base station are synchronized ${ }^{4}$. An illustration of the scenario is depicted in Fig. 1. The base station and

\footnotetext{
${ }^{2}$ Note that if a larger object is illuminated by the base station and multiple paths are reflected towards the mobile terminal, each path will contribute a distinct rank one matrix to the EFIM given that the paths are resolvable in the temporal or angular domain.

${ }^{3}$ Since Fisher information is a multidimensional concept, information can be represented as eigenvalue-eigenvector pairs of the (E)FIM [2], [3]. We refer to the eigenvalue as the gain of information and the angle of the eigenvector as the direction of information.

${ }^{4}$ The synchronization assumption can be eliminated by considering a twoway protocol [28], [29].
} 


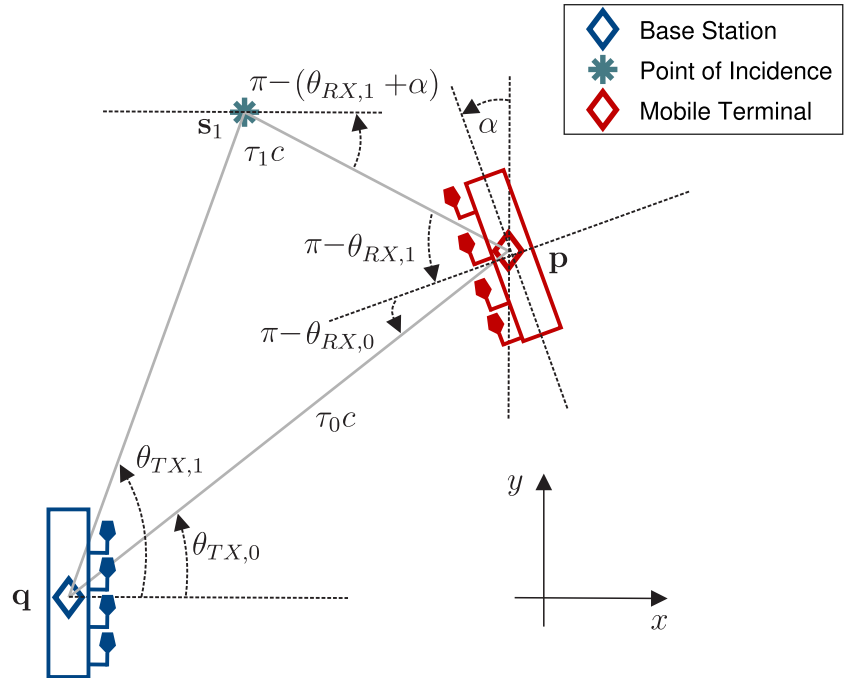

Fig. 1: Geometry of the scenario - A mobile terminal with unknown position and orientation attempts to localize itself and determine its orientation using the signal received from a base station. The base station has known location and orientation. Single-bounce NLOS paths and a direct path are considered.

mobile terminal are equipped with an array of $N_{\mathrm{TX}}$ transmit antennas and $N_{\mathrm{RX}}$ receive antennas, respectively. The array of the base station has arbitrary but known geometry. We denote the orientation of the base station array by $\phi$. The centroids of the base station and mobile terminal arrays are located at the positions $\mathbf{q}=\left[q_{\mathrm{x}}, q_{\mathrm{y}}\right]^{\mathrm{T}}$ and $\mathbf{p}=\left[p_{\mathrm{x}}, p_{\mathrm{y}}\right]^{\mathrm{T}}$, respectively. We assume that the array geometry of the mobile terminal is known while the orientation $\alpha$ of the array is unknown.

\section{B. Transmitter Model}

We consider mmWave in combination with massive MIMO. In particular, the transmitter transmits $\tilde{\mathbf{s}}(t) \triangleq \sqrt{E_{\mathbf{s}}} \mathbf{F} \mathbf{s}(t)$, where $E_{\mathrm{s}}$ denotes the energy per symbol, $\mathbf{F} \triangleq\left[\mathbf{f}_{1}, \mathbf{f}_{2}, \ldots, \mathbf{f}_{N_{\mathrm{B}}}\right]$ is a precoding matrix with $N_{\mathrm{B}}$ transmitted beams, and $\mathbf{s}(t) \triangleq$ $\left[s_{1}(t), \ldots, s_{N_{\mathrm{B}}}(t)\right]^{\mathrm{T}}$ is the vector of pilot signals. The pilot signal of the $l^{\text {th }}$ beam is given by

$$
s_{l}(t) \triangleq \sum_{m=0}^{N_{s}-1} d_{l, m} p\left(t-m T_{\mathrm{s}}\right),
$$

where $N_{s}$ denotes the number of pilot symbols per beam, $T_{s}$ is the symbol duration, $d_{l, m}$ the $m^{\text {th }}$ unit energy pilot symbol which is transmitted over the $l^{\text {th }}$ beam with the unit energy pulse $p(t)$. We assume that the pilot symbols $d_{l, m}, \forall l, m$ are independent and identically distributed (IID) with zero mean and known to the receiver. The $l^{\text {th }}$ column of $\mathbf{F}$ contains a directional beam pointing towards the azimuth angle $\theta_{\mathrm{BF}, l}$

$$
\mathbf{f}_{l}\left(\theta_{\mathrm{BF}, l}\right) \triangleq \frac{1}{\sqrt{N_{\mathrm{B}}}} \mathbf{a}_{\mathrm{TX}, l}\left(\theta_{\mathrm{BF}, l}\right),
$$

here, $\mathbf{a}_{\mathrm{TX}, l}$ is the unit-norm array response vector given by [30]

$$
\mathbf{a}_{\mathrm{TX}, l}\left(\theta_{\mathrm{BF}, l}\right) \triangleq \frac{1}{\sqrt{N_{\mathrm{TX}}}} \exp \left(-j \boldsymbol{\Delta}_{\mathrm{TX}}^{\mathrm{T}} \mathbf{k}\left(\theta_{\mathrm{BF}, l}\right)\right),
$$

where $\mathbf{k}\left(\theta_{\mathrm{BF}, l}\right)=\frac{2 \pi}{\lambda}\left[\cos \left(\theta_{\mathrm{BF}, l}\right), \sin \left(\theta_{\mathrm{BF}, l}\right)\right]^{\mathrm{T}}$ is the wavenumber vector, $\lambda$ is the wavelength, $\boldsymbol{\Delta}_{\mathrm{TX}} \triangleq$ $\left[\mathbf{u}_{\mathrm{TX}, 1}, \mathbf{u}_{\mathrm{TX}, 2}, \ldots, \mathbf{u}_{\mathrm{TX}, N_{\mathrm{TX}}}\right]$ is a $2 \times N_{\mathrm{TX}}$ matrix which contains the positions of the transmit antenna elements in $2 \mathrm{D}$ Cartesian coordinates in its columns, i.e. the $n^{\text {th }}$ column of $\boldsymbol{\Delta}_{\mathrm{TX}}$ is given by $\mathbf{u}_{\mathrm{TX}, n} \triangleq\left[x_{\mathrm{TX}, n}, y_{\mathrm{TX}, n}\right]^{\mathrm{T}}$. To normalize the transmitted power, we set $\operatorname{tr}\left(\mathbf{F}^{\mathrm{H}} \mathbf{F}\right)=1$ and $\mathbb{E}\left\{\mathbf{s}(t) \mathbf{s}(t)^{\mathrm{H}}\right\}=$ $\mathbf{I}_{N_{\mathrm{B}}}$, where $\mathbf{I}_{N_{\mathrm{B}}}$ is the $N_{\mathrm{B}}$-dimensional identity matrix.

\section{Channel Model}

We assume $K \geq 1$ distinct paths between the base station and the mobile terminal. When using mmWave massive MIMO, the number of paths is small [9]. The LOS path - if it exists - is denoted by $k=0$, while $k>0$ correspond to NLOS components. Note that we assume perfect knowledge regarding the presence of an LOS path. Due to the large path loss in the mmWave band, the received power of NLOS paths is significantly lower than that of the LOS path [31], and the LOS path can be detected reliably. In addition, due to the high path loss and the high directionality of the transmitted beams, NLOS components are assumed to originate from single-bounce scattering or reflection only [1], [18], [19], [32]. Scatterers are objects that are much smaller than the wavelength of the signal, while reflectors are objects with a specific reflection point that are much larger than the wavelength of the signal. We denote the reflecting point and the location of the scatterer by the point of incidence $\mathbf{s}_{k}=\left[s_{\mathrm{x}, k}, s_{\mathrm{y}, k}\right]^{\mathrm{T}}$. Considering Fig. 1, it can be seen that each path is associated with three distinct channel parameters, namely AOA, AOD, and TOA, where AOA, AOD, and TOA of the $k^{\text {th }}$ path are denoted by $\theta_{\mathrm{RX}, k}, \theta_{\mathrm{TX}, k}$, and $\tau_{k}$, respectively. Assuming a narrow-band array model ${ }^{5}$, the channel impulse response is given by

$$
\begin{aligned}
\mathbf{H}(t)=\sum_{k=0}^{K-1} & \underbrace{\sqrt{N_{\mathrm{RX}} N_{\mathrm{TX}}} h_{k} \mathbf{a}_{\mathrm{RX}, k}\left(\theta_{\mathrm{RX}, k}\right) \mathbf{a}_{\mathrm{TX}, k}^{H}\left(\theta_{\mathrm{TX}, k}\right)}_{\mathbf{H}_{k}} \\
& \times \delta\left(t-\tau_{k}\right),
\end{aligned}
$$

where $h_{k}=h_{\mathrm{R}, k}+j h_{\mathrm{I}, k}$ is the complex path gain while $\mathbf{a}_{\mathrm{TX}, k}\left(\theta_{\mathrm{TX}, k}\right)$ and $\mathbf{a}_{\mathrm{RX}, k}\left(\theta_{\mathrm{RX}, k}\right)$ denote the unit-norm array response vectors of the $k^{\text {th }}$ path at the transmitter and receiver, respectively. We emphasize that $\theta_{\mathrm{BF}, l}$ determines the angle of $l^{\text {th }}$ transmitted beam, while $\theta_{\mathrm{TX}, k}$ describes the angle between base station and the $k^{\text {th }}$ point of incidence. Note that $\mathbf{a}_{\mathrm{TX}, k}\left(\theta_{\mathrm{TX}, k}\right)$ is explicitly defined in (3), while $\mathbf{a}_{\mathrm{RX}, k}\left(\theta_{\mathrm{RX}, k}\right)$ can be defined analogously by (3) with appropriate subscripts. Bear in mind that paths may have angular spreads which can be included in the estimation problem [33]. However, no angular spreads are considered in this work.

\section{Receiver Model}

The noisy observed signal at the receiver is given by

$\mathbf{r}(t) \triangleq \sum_{k=0}^{K-1} \sqrt{E_{\mathbf{s}}} \mathbf{H}_{k} \mathbf{F} \mathbf{s}\left(t-\tau_{k}\right)+\mathbf{n}(t), \quad t \in\left[0, N_{s} T_{s}\right]$

${ }^{5}$ We assume that $A_{\max }<<c / B$, where $A_{\max }$ is the maximum array aperture size, $c$ is the speed of light, and $B$ is the system bandwidth. 
where $\mathbf{n}(t)=\left[n_{1}(t), n_{2}(t), \ldots, n_{N_{\mathrm{RX}}}(t)\right]^{\mathrm{T}}$ is zero-mean additive white Gaussian noise (AWGN) with power spectral density (PSD) $N_{0}$. Similar to [34], [35], we assume that a low-noise amplifier and a passband filter are attached to every receive antenna. This assumption might seem restrictive for the practical application, yet it simplifies the analysis of the EFIM. It can be regarded as the receiver architecture which results in the lowest $\mathrm{PEB}$ and OEB.

\section{E. System-Level Aspects}

In this paper, localization is considered in the downlink. In the uplink, the orientation of the mobile terminal influences the positioning accuracy. In certain scenarios, similar results can be obtained when compared to the downlink. A comprehensive comparison of uplink and downlink localization in $5 \mathrm{G}$ is considered in [20].

Localization can be performed during the initial access or the in-service phase. During the initial access phase, the environment is typically scanned by multiple beams in order to identify suitable beam patterns [36]. In this phase, no additional information regarding the location of the mobile terminal or the scatterers or reflectors is necessary to perform positioning. Only the multipath components of the reflectors or scatterers which are illuminated by these beams are received by the mobile terminal. From these components, the positions of the respective reflectors or scatterers can be estimated and stored in a map. If this map is known to the base station during the in-service phase, the beamforming vectors can be chosen such that the scatterers or reflectors are illuminated in order to increase the positioning accuracy of the mobile terminal or the capacity of the link.

\section{FISHER INFORMATION MATRIX OF THE CHANNEL PARAMETERS}

In this section, we first define the estimation problem and state the FIM of the channel parameters. We conclude the section with a brief summary of the results from [20], which allow for a simplification of the FIM of the channel parameters.

\section{A. Definition}

We first define the vector of channel parameters

$$
\boldsymbol{\eta} \triangleq\left[\boldsymbol{\theta}_{\mathrm{RX}}^{\mathrm{T}}, \boldsymbol{\theta}_{\mathrm{TX}}^{\mathrm{T}}, \boldsymbol{\tau}^{\mathrm{T}}, \mathbf{h}_{\mathrm{R}}^{\mathrm{T}}, \mathbf{h}_{\mathrm{I}}^{\mathrm{T}}\right]^{\mathrm{T}},
$$

where we collect the AOAs, AODs, TOAs, and channel gains in the vectors $\boldsymbol{\theta}_{\mathrm{RX}} \triangleq\left[\theta_{\mathrm{RX}, 0}, \theta_{\mathrm{RX}, 1}, \ldots, \theta_{\mathrm{RX}, K-1}\right]^{\mathrm{T}}, \boldsymbol{\theta}_{\mathrm{TX}} \triangleq$ $\left[\theta_{\mathrm{TX}, 0}, \theta_{\mathrm{TX}, 1}, \ldots, \theta_{\mathrm{TX}, K-1}\right]^{\mathrm{T}}, \boldsymbol{\tau} \triangleq\left[\tau_{0}, \tau_{1}, \ldots, \tau_{K-1}\right]^{\mathrm{T}}, \mathbf{h}_{\mathrm{R}} \triangleq$ $\left[h_{\mathrm{R}, 0}, h_{\mathrm{R}, 1}, \ldots, h_{\mathrm{R}, K-1}\right]^{\mathrm{T}}$, and $\mathbf{h}_{\mathrm{I}} \triangleq\left[h_{\mathrm{I}, 0}, h_{\mathrm{I}, 1}, \ldots, h_{\mathrm{I}, K-1}\right]^{\mathrm{T}}$, respectively. The corresponding FIM is given by

$$
\mathbf{J}_{\boldsymbol{\eta}} \triangleq\left[\begin{array}{cccc}
\mathbf{J}_{\boldsymbol{\theta}_{\mathrm{RX}} \boldsymbol{\theta}_{\mathrm{RX}}} & \mathbf{J}_{\boldsymbol{\theta}_{\mathrm{RX}} \boldsymbol{\theta}_{\mathrm{TX}}} & \cdots & \mathbf{J}_{\boldsymbol{\theta}_{\mathrm{RX}} \mathbf{h}_{\mathrm{I}}} \\
\mathbf{J}_{\boldsymbol{\theta}_{\mathrm{RX}}^{\mathrm{T}} \boldsymbol{\theta}_{\mathrm{TX}}}^{\mathrm{T}} & \ddots & \cdots & \vdots \\
\vdots & \cdots & \ddots & \vdots \\
\mathbf{J}_{\boldsymbol{\theta}_{\mathrm{RX}} \mathbf{h}_{\mathrm{I}}}^{\mathrm{T}} & \cdots & \cdots & \mathbf{J}_{\mathbf{h}_{\mathrm{I}} \mathbf{h}_{\mathrm{I}}}
\end{array}\right]
$$

where each entry of the FIM of the channel parameters can be computed according to ${ }^{6}$ [37]

$$
\left[\mathbf{J}_{\boldsymbol{\eta}}\right]_{u, v} \triangleq \frac{1}{N_{0}} \int_{0}^{N_{\mathrm{s}} T_{\mathrm{s}}} \mathbb{E}_{\mathrm{a}}\left[\Re\left\{\frac{\partial \boldsymbol{\mu}_{\boldsymbol{\eta}}^{\mathrm{H}}(t)}{\partial[\boldsymbol{\eta}]_{u}} \frac{\partial \boldsymbol{\mu}_{\boldsymbol{\eta}}(t)}{\partial[\boldsymbol{\eta}]_{v}}\right\}\right] \mathrm{d} t .
$$

In (8), $\mathbb{E}_{\mathrm{a}}[\cdot]$ denotes the expectation with respect to the pilot symbols, $\mathfrak{R}\{\cdot\}$ is the real part of the argument, and $\boldsymbol{\mu}_{\boldsymbol{\eta}}(t)$ is defined as the noise-free observation which can be obtained from (5) as

$$
\boldsymbol{\mu}_{\boldsymbol{\eta}}(t)=\sum_{k=0}^{K-1} \sqrt{E_{s}} \mathbf{H}_{k} \mathbf{F} \mathbf{s}\left(t-\tau_{k}\right) .
$$

The FIM is related to the estimation error covariance matrix of any unbiased estimator via the information inequality [38][40]

$$
\mathbb{E}\left[(\boldsymbol{\eta}-\hat{\boldsymbol{\eta}})(\boldsymbol{\eta}-\hat{\boldsymbol{\eta}})^{\mathrm{T}}\right] \succeq \mathbf{J}_{\boldsymbol{\eta}}^{-1}
$$

where $\hat{\boldsymbol{\eta}}$ is the estimate of $\boldsymbol{\eta}$ and $\mathbf{A} \succeq \mathbf{B}$ is equivalent to $\mathbf{A}-\mathbf{B}$ being positive semi-definite. The inequality in (10) is the well-known Cramér-Rao lower bound (CRLB).

\section{B. Simplification}

The blocks of the FIM in (7) obey certain scaling laws when the number of receive and transmit antennas, as well as the bandwidth become sufficiently large ${ }^{7}$. In particular, it was shown in [20, Section III-B] that some blocks can be well approximated by diagonal matrices, while others become zero matrices. In the following, we provide a brief summary of the results from [20].

Let $\mathbf{I}_{\mathrm{K}}$ and $\mathbf{0}_{\mathrm{K}}$ be the $K \times K$ identity and all-zeros matrix, respectively. We denote the Hadamard product by $\odot$, and make the following remarks:

1) Since the AOAs of the different paths are assumed to be distinct, the steering vectors at the receiver do not interact considerably with each other, i.e. $\left\|\mathbf{a}_{\mathrm{RX}, u}^{\mathrm{H}} \mathbf{a}_{\mathrm{RX}, v}\right\| \ll$ $\left\|\mathbf{a}_{\mathrm{RX}, u}^{\mathrm{H}} \mathbf{a}_{\mathrm{RX}, u}^{\mathrm{H}}\right\|, u \neq v$. Hence AOAs can be estimated independently, i.e. $\tilde{\mathbf{J}}_{\boldsymbol{\theta}_{\mathrm{RX}} \boldsymbol{\theta}_{\mathrm{RX}}} \approx \mathbf{I}_{\mathrm{K}} \odot \mathbf{J}_{\boldsymbol{\theta}_{\mathrm{RX}} \boldsymbol{\theta}_{\mathrm{RX}}}$.

2) The transmitted beams have no spatial cross-correlation and hence AODs can be estimated independently, i.e. $\tilde{\mathbf{J}}_{\boldsymbol{\theta}_{\mathrm{TX}} \boldsymbol{\theta}_{\mathrm{TX}}} \approx \mathbf{I}_{\mathrm{K}} \odot \mathbf{J}_{\boldsymbol{\theta}_{\mathrm{TX}} \boldsymbol{\theta}_{\mathrm{TX}}}$.

3) The NLOS cross-correlation vanishes as the bandwidth of the signal becomes large since the paths can be resolved independently in time, i.e. $\tilde{\mathbf{J}}_{\boldsymbol{\tau} \boldsymbol{\tau}} \approx \mathbf{I}_{\mathrm{K}} \odot \mathbf{J}_{\boldsymbol{\tau} \boldsymbol{\tau}}$.

4) As a consequence of the previous results, the channel gains can be estimated independently, i.e. $\tilde{\mathbf{J}}_{\mathbf{h}_{\mathrm{R}} \mathbf{h}_{\mathrm{R}}} \approx \mathbf{I}_{\mathrm{K}} \odot$ $\mathbf{J}_{\mathbf{h}_{\mathrm{R}} \mathbf{h}_{\mathrm{R}}}$ and $\tilde{\mathbf{J}}_{\mathbf{h}_{\mathrm{I}} \mathbf{h}_{\mathrm{I}}} \approx \mathbf{I}_{\mathrm{K}} \odot \mathbf{J}_{\mathbf{h}_{\mathrm{I}} \mathbf{h}_{\mathrm{I}}}$.

5) All off-diagonal blocks, except for $\mathbf{J}_{\boldsymbol{\theta}_{\mathrm{TX}} \mathbf{h}_{\mathrm{R}}}$ and $\mathbf{J}_{\boldsymbol{\theta}_{\mathrm{TX}} \mathbf{h}_{\mathrm{I}}}$, in (7) become zero. It was shown in [20] that the real and imaginary parts of the $k^{\text {th }}$ channel gain couple only with the AOD of the $k^{\text {th }}$ path, i.e. $\tilde{\mathbf{J}}_{\boldsymbol{\theta}_{\mathrm{TX}} \mathbf{h}_{\mathrm{R}}} \approx \mathbf{I}_{\mathrm{K}} \odot \mathbf{J}_{\boldsymbol{\theta}_{\mathrm{TX}} \mathbf{h}_{\mathrm{R}}}$ and $\tilde{\mathbf{J}}_{\boldsymbol{\theta}_{\mathrm{TX}} \mathbf{h}_{\mathrm{I}}} \approx \mathbf{I}_{\mathrm{K}} \odot \mathbf{J}_{\boldsymbol{\theta}_{\mathrm{TX}} \mathbf{h}_{\mathrm{I}}}$.

\footnotetext{
${ }^{6}$ This result holds whenever the signal is observed under AWGN.

${ }^{7}$ It was shown numerically in [20] that the approximation error of the PEB due to the simplification of the FIM is fairly small even under realistic assumption on the bandwidth $(B=125 \mathrm{MHz})$ and the $2 \mathrm{D}$ array sizes $\left(N_{\mathrm{TX} / \mathrm{RX}}=12 \times 12\right)$.
} 
Thus, when the bandwidth of the signal is large and number of receive and transmit antennas is also large, $\mathbf{J}_{\boldsymbol{\eta}}$ can be well approximated by

$$
\tilde{\mathbf{J}}_{\boldsymbol{\eta}} \triangleq\left[\begin{array}{ccccc}
\tilde{\mathbf{J}}_{\boldsymbol{\theta}_{\mathrm{RX}} \boldsymbol{\theta}_{\mathrm{RX}}} & \mathbf{0}_{\mathrm{K}} & \mathbf{0}_{\mathrm{K}} & \mathbf{0}_{\mathrm{K}} & \mathbf{0}_{\mathrm{K}} \\
\mathbf{0}_{\mathrm{K}} & \tilde{\mathbf{J}}_{\boldsymbol{\theta}_{\mathrm{TX}} \boldsymbol{\theta}_{\mathrm{TX}}} & \mathbf{0}_{\mathrm{K}} & \tilde{\mathbf{J}}_{\boldsymbol{\theta}_{\mathrm{TX}} \mathbf{h}_{\mathrm{R}}} & \tilde{\mathbf{J}}_{\boldsymbol{\theta}_{\mathrm{TX}} \mathbf{h}_{\mathrm{I}}} \\
\mathbf{0}_{\mathrm{K}} & \mathbf{0}_{\mathrm{K}} & \tilde{\mathbf{J}}_{\boldsymbol{\tau} \boldsymbol{\tau}} & \mathbf{0}_{\mathrm{K}} & \mathbf{0}_{\mathrm{K}} \\
\mathbf{0}_{\mathrm{K}} & \tilde{\mathbf{J}}_{\boldsymbol{\theta}_{\mathrm{T}} \mathbf{h}_{\mathrm{R}}}^{\mathrm{T}} & \mathbf{0}_{\mathrm{K}} & \tilde{\mathbf{J}}_{\mathbf{h}_{\mathrm{R}} \mathbf{h}_{\mathrm{R}}} & \mathbf{0}_{\mathrm{K}} \\
\mathbf{0}_{\mathrm{K}} & \tilde{\mathbf{J}}_{\boldsymbol{\theta}_{\mathrm{TX}} \mathbf{h}_{\mathrm{I}}}^{\mathrm{T}} & \mathbf{0}_{\mathrm{K}} & \mathbf{0}_{\mathrm{K}} & \tilde{\mathbf{J}}_{\mathbf{h}_{\mathrm{I}} \mathbf{h}_{\mathrm{I}}}
\end{array}\right] .
$$

\section{FISHER INFORMATION MATRIX OF THE POSITION-RELATED PARAMETERS}

Motivated by the findings discussed in the previous subsection, we first reorder the parameters of the simplified FIM $\tilde{\mathbf{J}}_{\boldsymbol{\eta}}$ in (11). Subsequently, we transform the resulting FIM to the position, orientation, and point of incidence domain. Then, we determine the EFIM of the position and orientation, which we decompose to analyze the impact of the LOS and NLOS paths.

\section{A. Derivation of the EFIM}

For mathematical convenience, we reorder the parameter vector $\boldsymbol{\eta}$ as follows

$$
\tilde{\boldsymbol{\eta}} \triangleq\left[\tilde{\boldsymbol{\eta}}_{0}^{\mathrm{T}}, \ldots, \tilde{\boldsymbol{\eta}}_{K-1}^{\mathrm{T}}\right]^{\mathrm{T}},
$$

where $\tilde{\boldsymbol{\eta}}_{k} \triangleq\left[\tau_{k}, \theta_{\mathrm{TX}, k}, h_{\mathrm{R}, \mathrm{k}}, h_{\mathrm{I}, \mathrm{k}}, \theta_{\mathrm{RX}, k}\right]^{\mathrm{T}}$. Reordering the parameter vector of the FIM results in a permutation of the entries of the FIM in (11). The reordered FIM is given by

$$
\mathbf{J}_{\tilde{\eta}} \triangleq \mathbf{P}_{\pi} \tilde{\mathbf{J}}_{\boldsymbol{\eta}}
$$

where $\mathbf{P}_{\pi}$ is a permutation matrix of size $5 K \times 5 K$ which is given by

$$
\mathbf{P}_{\pi} \triangleq\left[\mathbf{P}_{\pi, 1}, \ldots, \mathbf{P}_{\pi, K}\right]^{\mathrm{T}}
$$

where $\mathbf{P}_{\pi, i}=\left[\mathbf{e}_{2 K+i}, \mathbf{e}_{K+i}, \mathbf{e}_{3 K+i}, \mathbf{e}_{4 K+i}\right]$ and $\mathbf{e}_{k}$ denotes the $k^{\text {th }}$ unit vector of the standard basis of appropriate length. Hence the FIM of the channel parameters has the following structure

$$
\mathbf{J}_{\tilde{\boldsymbol{\eta}}}=\operatorname{blkdiag}\left(\mathbf{J}_{\breve{\eta}_{0}}, \ldots, \mathbf{J}_{\breve{\eta}_{K-1}}\right) \text {. }
$$

The FIM block $\mathbf{J}_{\tilde{\boldsymbol{\eta}}_{k}}$ of the $k^{\text {th }}$ path is given by

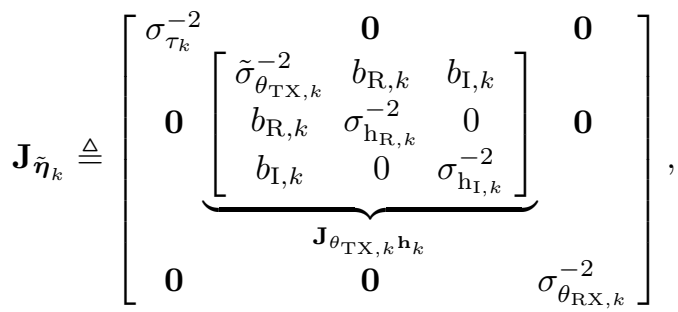

where we abbreviate the diagonal entries of the reordered FIM matrix of the channel parameters by the respective $\sigma^{-2}$ terms, while $b_{\mathrm{R}, k}$ and $b_{\mathrm{I}, k}$ denote the corresponding entries of $\tilde{\mathbf{J}}_{\boldsymbol{\theta}_{\mathrm{TX}} \mathbf{h}_{\mathrm{R}}}$ and $\tilde{\mathbf{J}}_{\boldsymbol{\theta}_{\mathrm{TX}} \mathbf{h}_{\mathrm{I}}}$, respectively. Since, for position and orientation estimation, we are mainly interested in the triplets of AOA, AOD, and TOA of every path, we combine the uncertainty of $h_{\mathrm{R}, \mathrm{k}}$ and $h_{\mathrm{I}, \mathrm{k}}$ in the AOD-related term. For that, we will use the notion of the EFIM to consider only the information concerning AOA, AOD, and TOA. The EFIM is a measure of the information corresponding to certain parameters, while accounting for the uncertainties of other (unknown) parameters.

Definition 1. Given a parameter vector $\boldsymbol{\xi} \triangleq\left[\boldsymbol{\xi}_{1}^{\mathrm{T}}, \boldsymbol{\xi}_{2}^{\mathrm{T}}\right]^{\mathrm{T}}$ with corresponding FIM

$$
\mathbf{J}_{\boldsymbol{\xi}} \triangleq\left[\begin{array}{ll}
\mathbf{J}_{\xi_{1} \xi_{1}} & \mathbf{J}_{\boldsymbol{\xi}_{1} \boldsymbol{\xi}_{2}} \\
\mathbf{J}_{\boldsymbol{\xi}_{1}}^{\mathrm{T}} \boldsymbol{\xi}_{2} & \mathbf{J}_{\boldsymbol{\xi}_{2} \boldsymbol{\xi}_{2}}
\end{array}\right]
$$

the EFIM of $\boldsymbol{\xi}_{1}$ is obtained by [41]

$$
\mathbf{J}_{\boldsymbol{\xi}_{1}}^{\mathrm{e}} \triangleq \mathbf{J}_{\boldsymbol{\xi}_{1} \boldsymbol{\xi}_{1}}-\mathbf{J}_{\boldsymbol{\xi}_{1} \boldsymbol{\xi}_{2}} \mathbf{J}_{\boldsymbol{\xi}_{2} \boldsymbol{\xi}_{2}}^{-1} \mathbf{J}_{\boldsymbol{\xi}_{1} \boldsymbol{\xi}_{2}}^{\mathrm{T}} \text {. }
$$

Intuitively, the fact that the parameters of $\boldsymbol{\xi}_{2}$ are not perfectly known, leads to a loss in information which is quantified by $\mathbf{J}_{\xi_{1} \xi_{2}} \mathbf{J}_{\boldsymbol{\xi}_{2} \boldsymbol{\xi}_{2}}^{-1} \mathbf{J}_{\boldsymbol{\xi}_{1} \boldsymbol{\xi}_{2}}^{\mathrm{T}}$.

Using (16) and (18), the EFIM of $\mathbf{J}_{\theta_{\mathrm{TX}, k}} \mathbf{h}_{k}$ with respect to $\theta_{\mathrm{TX}, k}$ is given by

$$
\mathbf{J}_{\theta_{\mathrm{TX}, k}}^{\mathrm{e}}=\frac{1}{\tilde{\sigma}_{\theta_{\mathrm{TX}, \mathrm{k}}}^{2}}-\left(b_{\mathrm{R}, \mathrm{k}}^{2} \sigma_{h_{\mathrm{R}, \mathrm{k}}}^{2}+b_{\mathrm{I}, \mathrm{k}}^{2} \sigma_{h_{\mathrm{I}, \mathrm{k}}}^{2}\right) \triangleq \frac{1}{\sigma_{\theta_{\mathrm{TX}, \mathrm{k}}}^{2}} .
$$

Hence the $(\mathrm{E}) \mathrm{FIM}^{8}$ of the $k^{\text {th }}$ path is given by $\mathbf{J}_{\overline{\boldsymbol{\eta}}_{k}} \triangleq \operatorname{diag}\left(1 / \sigma_{\tau_{\mathrm{k}}}^{2}, 1 / \sigma_{\theta_{\mathrm{TX}, \mathrm{k}}}^{2}, 1 / \sigma_{\theta_{\mathrm{RX}, \mathrm{k}}}^{2}\right)$, where $\overline{\boldsymbol{\eta}}_{k}=\left[\tau_{\mathrm{k}}, \theta_{\mathrm{TX}, \mathrm{k}}, \theta_{\mathrm{RX}, \mathrm{k}}\right]^{\mathrm{T}}$. For notational convenicence, we define $\mathbf{J}_{\overline{\boldsymbol{\eta}}_{\mathrm{LOS}}} \triangleq \mathbf{J}_{\overline{\boldsymbol{\eta}}_{0}}$ and $\mathbf{J}_{\overline{\boldsymbol{\eta}}_{\mathrm{NLOS}}} \triangleq \operatorname{blkdiag}\left(\mathbf{J}_{\overline{\boldsymbol{\eta}}_{1}}, \ldots, \mathbf{J}_{\overline{\boldsymbol{\eta}}_{K-1}}\right)$. Consequently, the FIM of all paths is given by

$$
\mathbf{J}_{\bar{\eta}} \triangleq \operatorname{blkdiag}\left(\mathbf{J}_{\overline{\boldsymbol{\eta}}_{\mathrm{LOS}}}, \mathbf{J}_{\overline{\boldsymbol{\eta}}_{\mathrm{NLOS}}}\right) \text {. }
$$

Each $\sigma^{2}$-term reflects the quality of the parameter estimation of the respective parameter, e.g., large $\sigma_{\tau_{0}}^{2}$ means that the TOA of the first path cannot be estimated accurately. Note that the $\sigma^{2}$-terms in (20) depend on the number of antennas, beamforming, bandwidth, and receiver location, as evaluated and analyzed in [20]. Note that our model assumes specular reflection or scattering. However, if paths are close in angle and delay, then they should be merged into a single path with the sum of the complex gains or modeled as a cluster. The Fisher information which is contributed by such a sum of similar paths or a cluster would be smaller than the information from a path with specular reflection or scattering. To account for this, additional measurement noise can be considered, i.e. the $\sigma^{2}$-terms in (16) can be increased.

Finally, we transform the FIM in (20) to the position, orientation, and point of incidence domain using the geometric relationships between the channel parameters $\overline{\boldsymbol{\eta}}=\left[\overline{\boldsymbol{\eta}}_{0}, \ldots, \overline{\boldsymbol{\eta}}_{K-1}\right]$ and $\breve{\boldsymbol{\eta}} \triangleq\left[\mathbf{p}^{\mathrm{T}}, \alpha, \mathbf{s}_{1}^{\mathrm{T}}, \ldots, \mathbf{s}_{K-1}^{\mathrm{T}}\right]^{\mathrm{T}}$. In particular, the FIM of the position-related parameters is given by [37]

$$
\mathbf{J}_{\breve{\eta}} \triangleq \mathbf{T} \mathbf{J}_{\bar{\eta}} \mathbf{T}^{\mathrm{T}}
$$

where $\mathbf{T} \triangleq \frac{\partial \overline{\boldsymbol{\eta}}^{\mathrm{T}}}{\partial \overline{\boldsymbol{\eta}}}$.

Remark: We normalize the units of the position-related and orientation-related entries in the transformation matrix by 1 $\mathrm{m}$ and $1 \mathrm{rad}$, respectively, in order to avoid a mixed-units FIM of the position, orientation, and points of incidence after the transformation. After the (normalized) transformation, the

\footnotetext{
${ }^{8}$ We slightly abuse the notation here since we denote the EFIM $\mathbf{J}_{\overline{\boldsymbol{\eta}}_{k}}^{\mathrm{e}}$ by $\mathbf{J}_{\bar{\eta}_{k}}$ and call it FIM to avoid confusion with another EFIM later on.
} 


$$
\mathbf{\Upsilon}_{n, m}(\theta, \phi, \rho) \triangleq\left[\begin{array}{ccc}
\cos ^{2}(\theta+\phi) & (-1)^{n} \sin (\theta) \cos (\theta) & (-1)^{m} \rho \sin (\theta) \\
(-1)^{n} \sin (\theta) \cos (\theta) & \sin ^{2}(\theta+\phi) & (-1)^{m+1} \rho \cos (\theta) \\
(-1)^{m} \rho \sin (\theta) & (-1)^{m+1} \rho \cos (\theta) & \rho^{2}
\end{array}\right]
$$

FIM of the position, orientation, and points of incidence will be dimensionless, which allows us to employ the standard inner product (dot product) to define the norm of a vector. For notational convenience, we omit the normalization constants (1 $\mathrm{m}$ and $1 \mathrm{rad})$.

Lemma 1. The transformation matrix $\mathbf{T}$ is an upper triangle block-matrix

$$
\mathbf{T} \triangleq\left[\begin{array}{c|ccc}
\mathbf{T}_{\mathbf{P}}^{(0)} & \mathbf{T}_{\mathbf{P}}^{(1)} & \cdots & \mathbf{T}_{\mathbf{P}}^{(K-1)} \\
\hline \mathbf{0} & \mathbf{T}_{\mathbf{s}_{1}} & \cdots & \mathbf{0} \\
\vdots & \vdots & \ddots & \vdots \\
\mathbf{0} & \mathbf{0} & \cdots & \mathbf{T}_{\mathbf{s}_{K-1}}
\end{array}\right] \triangleq\left[\begin{array}{c|c}
\mathbf{A} & \mathbf{B} \\
\hline \mathbf{0} & \mathbf{D}
\end{array}\right],
$$

where [19]

$$
\mathbf{T}_{\mathbf{P}}^{(k)}=\left[\begin{array}{lll}
\partial \tau_{k} / \partial \mathbf{p} & \partial \theta_{\mathrm{TX}, k} / \partial \mathbf{p} & \partial \theta_{\mathrm{RX}, k} / \partial \mathbf{p} \\
\partial \tau_{k} / \partial \alpha & \partial \theta_{\mathrm{TX}, k} / \partial \alpha & \partial \theta_{\mathrm{RX}, k} / \partial \alpha
\end{array}\right]
$$

and

$$
\mathbf{T}_{\mathbf{s}_{k}}=\left[\begin{array}{lll}
\partial \tau_{k} / \partial \mathbf{s}_{k} & \partial \theta_{\mathrm{TX}, k} / \partial \mathbf{s}_{k} \partial \theta_{\mathrm{RX}, k} / \partial \mathbf{s}_{k}
\end{array}\right] .
$$

The individual elements are summarized in Appendix A.

Lemma 2. The FIM of the position-related parameters is given by

$$
\mathbf{J}_{\breve{\eta}}=\left[\begin{array}{c|c}
\mathbf{A} \mathbf{J}_{\overline{\boldsymbol{\eta}}_{\mathrm{LOS}}} \mathbf{A}^{\mathrm{T}}+\mathbf{B} \mathbf{J}_{\overline{\boldsymbol{\eta}}_{\mathrm{NLOS}}} \mathbf{B}^{\mathrm{T}} & \mathbf{B} \mathbf{J}_{\overline{\boldsymbol{\eta}}_{\mathrm{NLOS}}} \mathbf{D}^{\mathrm{T}} \\
\hline \mathbf{D} \mathbf{J}_{\overline{\boldsymbol{\eta}}_{\mathrm{NLOS}}} \mathbf{B}^{\mathrm{T}} & \mathbf{D} \mathbf{J}_{\overline{\boldsymbol{\eta}}_{\mathrm{NLOS}}} \mathbf{D}^{\mathrm{T}}
\end{array}\right]
$$

Proof. Evaluate (21) using Lemma 1.

Defining $\breve{\boldsymbol{\eta}}_{\mathbf{p}, \alpha} \triangleq\left[\mathbf{p}^{\mathrm{T}}, \alpha\right]^{\mathrm{T}}$, we obtain the EFIM of the position and orientation using (18) and (26) from Lemma 2

$$
\begin{aligned}
& \mathbf{J}_{\tilde{\boldsymbol{\eta}}_{\mathbf{p}, \alpha}}^{\mathrm{e}}=\underbrace{\mathbf{A} \mathbf{J}_{\overline{\boldsymbol{\eta}}_{\mathrm{LOS}}} \mathbf{A}^{\mathrm{T}}}_{\triangleq \tilde{\mathbf{A}}^{(\mathrm{G})} \text { - LOS info gain }}+\underbrace{\mathbf{B} \mathbf{J}_{\overline{\boldsymbol{\eta}}_{\mathrm{NLOS}}} \mathbf{B}^{\mathrm{T}}}_{\triangleq \tilde{\mathbf{B}}^{(\mathrm{G})} \text { - NLOS info gain }} \\
& -\underbrace{\mathbf{B} \mathbf{J}_{\overline{\boldsymbol{\eta}}_{\mathrm{NLOS}}} \mathbf{D}^{\mathrm{T}}\left(\mathbf{D} \mathbf{J}_{\overline{\boldsymbol{\eta}}_{\mathrm{NLOS}}} \mathbf{D}^{\mathrm{T}}\right)^{-1} \mathbf{D} \mathbf{J}_{\overline{\boldsymbol{\eta}}_{\mathrm{NLOS}}} \mathbf{B}^{\mathrm{T}}}_{\triangleq \tilde{\mathbf{B}}^{(\mathrm{L})} \text { - NLOS info loss }} .
\end{aligned}
$$

It becomes evident that the EFIM is composed of three terms. The first term $\tilde{\mathbf{A}}^{(\mathrm{G})}$ quantifies the information gain from the LOS path. The second term $\tilde{\mathbf{B}}^{(\mathrm{G})}$ quantifies the information gain from the NLOS components. Finally, the third term $\tilde{\mathbf{B}}^{(\mathrm{L})}$ specifies the loss of information which accounts for the fact that the points of incident of NLOS paths are unknown. Considering the structure of $\mathbf{J}_{\bar{\eta}}$ and $\mathbf{T}$ the NLOS info gain and loss can be written as

$$
\tilde{\mathbf{B}}^{(\mathrm{G})}=\sum_{k=1}^{K-1} \mathbf{T}_{\mathbf{P}}^{(k)} \mathbf{J}_{\overline{\boldsymbol{\eta}}_{k}}\left(\mathbf{T}_{\mathbf{P}}^{(k)}\right)^{\mathrm{T}},
$$

and

$$
\begin{aligned}
\tilde{\mathbf{B}}^{(\mathrm{L})}=\sum_{k=1}^{K-1} & \mathbf{T}_{\mathbf{P}}^{(k)} \mathbf{J}_{\overline{\boldsymbol{\eta}}_{k}}\left(\mathbf{T}_{\mathbf{s}_{k}}\right)^{\mathrm{T}}\left(\mathbf{T}_{\mathbf{s}_{k}} \mathbf{J}_{\overline{\boldsymbol{\eta}}_{k}}\left(\mathbf{T}_{\mathbf{s}_{k}}\right)^{\mathrm{T}}\right)^{-1} \\
& \times \mathbf{T}_{\mathbf{s}_{k}} \mathbf{J}_{\overline{\boldsymbol{\eta}}_{k}}\left(\mathbf{T}_{\mathbf{P}}^{(k)}\right)^{\mathrm{T}}
\end{aligned}
$$

respectively.

Interpretation: The term in (28) reflects the gain of information from NLOS components if the positions of the corresponding points of incident $\mathbf{s}_{k}$ were perfectly known. Since the position of each point of incidence has to be estimated as well, the estimation uncertainty leads to a loss of information which is quantified in (29).

Outline: In the following, we decompose the terms $\mathbf{A}^{(\mathrm{G})}$ and $\mathbf{B}^{(\mathrm{N})}=\mathbf{B}^{(\mathrm{G})}-\mathbf{B}^{(\mathrm{L})}$ with the goal to express the EFIM in (27) as the sum of rank one matrices, where each rank one matrix is given by the outer-product of the unit-norm eigenvector $\mathbf{v}_{k}$ corresponding to the only non-zero eigenvalue $\lambda_{k}$ of the rank one matrix, i.e. $\mathbf{J}_{\grave{\eta}_{\mathbf{p}, \alpha}}^{\mathrm{e}}=\sum_{k} \lambda_{k} \mathbf{v}_{k} \mathbf{v}_{k}^{\mathrm{T}}$. We show that each rank one matrix corresponding to an NLOS path is positive semi-definite meaning that each NLOS path improves the position and orientation estimation accuracy. For notational convenience, we define the matrix template in (22) at the top of this page.

\section{B. LOS Information Gain}

Using (23), the results from Appendix A, and by simple algebra, the first term $\tilde{\mathbf{A}}^{(\mathrm{G})}$ in (27) can be easily shown to be

$$
\begin{aligned}
\tilde{\mathbf{A}}^{(\mathrm{G})}= & \underbrace{\frac{1}{\sigma_{\tau_{0}}^{2} c^{2}} \mathbf{\Upsilon}_{0,0}\left(\theta_{\mathrm{TX}, 0}, 0,0\right)}_{\tilde{\mathbf{A}}_{R}^{(\mathrm{G})}} \\
& +\underbrace{\frac{1}{\sigma_{\theta_{\mathrm{TX}, 0}^{2}}^{2}\|\mathbf{p}-\mathbf{q}\|^{2}} \mathbf{\Upsilon}_{1,0}\left(\theta_{\mathrm{TX}, 0}, \pi / 2,0\right)}_{\tilde{\mathbf{A}}_{D}^{(\mathrm{G})}} \\
& +\underbrace{\frac{1}{\sigma_{\theta_{\mathrm{RX}, 0}^{2}}^{2}\|\mathbf{p}-\mathbf{q}\|^{2}} \mathbf{\Upsilon}_{1,0}\left(\theta_{\mathrm{TX}, 0}, \pi / 2,\|\mathbf{p}-\mathbf{q}\|\right)}_{\tilde{\mathbf{A}}_{A}^{(\mathrm{G})}} .
\end{aligned}
$$

Proposition 1. The eigenvalues of the matrices $\tilde{\mathbf{A}}_{R}^{(G)}, \tilde{\mathbf{A}}_{D}^{(G)}$, and $\tilde{\mathbf{A}}_{A}^{(\mathrm{G})}$ are given by

$$
\begin{aligned}
\lambda_{R, 0}^{(\mathrm{G})} & =\frac{1}{\sigma_{\tau_{0}}^{2} c^{2}}, \\
\lambda_{D, 0}^{(\mathrm{G})} & =\frac{1}{\sigma_{\theta_{\mathrm{TX}, 0}}^{2}\|\mathbf{p}-\mathbf{q}\|^{2}}, \\
\lambda_{A, 0}^{(\mathrm{G})} & =\frac{\|\mathbf{p}-\mathbf{q}\|^{2}+1}{\sigma_{\theta_{\mathrm{RX}, 0}}^{2}\|\mathbf{p}-\mathbf{q}\|^{2}},
\end{aligned}
$$

respectively. The corresponding eigenvectors are given by

$$
\begin{aligned}
\mathbf{v}_{R, 0}^{(\mathrm{G})} & =\left[\cos \left(\theta_{\mathrm{TX}, 0}\right), \sin \left(\theta_{\mathrm{TX}, 0}\right), 0\right]^{\mathrm{T}}, \\
\mathbf{v}_{D, 0}^{(\mathrm{G})} & =\left[-\sin \left(\theta_{\mathrm{TX}, 0}\right), \cos \left(\theta_{\mathrm{TX}, 0}\right), 0\right]^{\mathrm{T}}, \\
\mathbf{v}_{A, 0}^{(\mathrm{G})} & =v_{A, 0}^{(\mathrm{G})}\left[\sin \left(\theta_{\mathrm{TX}, 0}\right),-\cos \left(\theta_{\mathrm{TX}, 0}\right),\|\mathbf{p}-\mathbf{q}\|\right]^{\mathrm{T}},
\end{aligned}
$$




\begin{tabular}{|c|c|c|c|c|}
\hline$\theta_{\mathrm{TX}, 0}$ & $\theta_{\mathrm{RX}, 0}$ & $\tau_{0}$ & $\mathbf{p}$ & $\alpha$ \\
\hline$\times$ & $\sqrt{ }$ & $\sqrt{ }$ & $\times$ & $\times$ \\
\hline$\sqrt{ }$ & $\times$ & $\sqrt{ }$ & $\sqrt{ }$ & $\times$ \\
\hline$\sqrt{ }$ & $\sqrt{ }$ & $\times$ & $\times$ & $\sqrt{ }$ \\
\hline
\end{tabular}

TABLE I: Relationship between the channel parameters $\theta_{\mathrm{TX}, 0}, \theta_{\mathrm{RX}, 0}$, $\tau_{0}$ and the identifiably of the position $\mathbf{p}$ and orientation $\alpha$. For instance ( $1^{\text {st }}$ row), if $\theta_{\mathrm{TX}, 0}$ cannot be estimated, neither the position $\mathbf{p}$ nor the orientation $\alpha$ can be identified.

where $v_{A, 0}^{(\mathrm{G})}=\sqrt{1 /\left(1+\|\mathbf{p}-\mathbf{q}\|^{2}\right)}$ ensures that $\mathbf{v}_{A, 0}^{(\mathrm{G})}$ is unit-norm. It is immediately obvious that all eigenvalues are positive.

Proof. See Appendix B.

Interpretation: TOA, AOD, and AOA of the LOS path contribute information to the EFIM. Each of these quantities provides information in one direction, where the direction is given by the eigenvector corresponding to the non-zero eigenvalue. When the variance of a channel parameter (e.g, $\left.\sigma_{\theta_{\mathrm{TX}, 0}}^{2}\right)$ is finite, information is contributed to the EFIM. For infinite variance, no information is contributed, i.e. the parameter is missing in the estimation problem. We summarize the cases where one parameter is missing in TABLE I, where ' $x$ ' means that the parameter is missing or cannot be determined and ' $\sqrt{ }$ ' means that the parameter is available or can be determined. Note that these observations can also be obtained from the equations which relate the channel parameters to the position $\left(\mathbf{p}=\mathbf{q}+\tau_{0} c\left[\cos \left(\theta_{\mathrm{TX}, 0}\right), \sin \left(\theta_{\mathrm{TX}, 0}\right)\right]^{T}\right)$ and the orientation $\left(\alpha=\pi+\theta_{\mathrm{TX}, 0}-\theta_{\mathrm{RX}, 0}\right)$. Considering (31b) and (31c), we see that the information gain reduces as the separation between base station and mobile terminal increases. For a large separation, AOA provides only orientation information since the $x-y$ components of the eigenvector in (32c) go to zero. In that case, the amount of orientation information is given by $1 / \sigma_{\theta_{\mathrm{RX}, 0}}^{2}$.

\section{NLOS Information Gain}

Similar to the previous section, we decompose the information matrices of NLOS paths using (23) and the results from Appendix A.

Lemma 3. The NLOS information gain matrix is given by

$$
\begin{aligned}
\tilde{\mathbf{B}}^{(\mathrm{G})}= & \sum_{k=1}^{K-1} \underbrace{\frac{1}{\sigma_{\tau_{k}}^{2} c^{2}} \boldsymbol{\Upsilon}_{0,0}\left(\theta_{\mathrm{RX}, k}, 0,0\right)}_{\tilde{\mathbf{B}}_{R, k}^{(\mathrm{G})}} . \\
& +\underbrace{\frac{1}{\sigma_{\theta_{\mathrm{RX}, k}}^{2}\left\|\mathbf{p}-\mathbf{s}_{k}\right\|^{2}} \mathbf{\Upsilon}_{1,1}\left(\theta_{\mathrm{RX}, k}, \pi / 2,\left\|\mathbf{p}-\mathbf{s}_{k}\right\|\right)}_{\tilde{\mathbf{B}}_{A, k}^{(\mathrm{G})}} .
\end{aligned}
$$

Proof. See Appendix C-A.

Note that the matrices $\tilde{\mathbf{B}}_{R, k}^{(\mathrm{G})}$ and $\tilde{\mathbf{B}}_{A, k}^{(\mathrm{G})}$ have the similar structure as $\tilde{\mathbf{A}}_{R}^{(\mathrm{G})}$ and $\tilde{\mathbf{A}}_{A}^{(\mathrm{G})}$, respectively. Hence the eigen- values and eigenvectors have similar structure as well, and are given by

$$
\begin{aligned}
\lambda_{R, k}^{(\mathrm{G})} & =\frac{1}{\sigma_{\tau_{k}}^{2} c^{2}}, \\
\lambda_{A, k}^{(\mathrm{G})} & =\frac{\left\|\mathbf{p}-\mathbf{s}_{k}\right\|^{2}+1}{\sigma_{\theta_{\mathrm{RX}, k}}^{2}\left\|\mathbf{p}-\mathbf{s}_{k}\right\|^{2}},
\end{aligned}
$$

and

$$
\begin{aligned}
& \mathbf{v}_{R, k}^{(\mathrm{G})}=\left[\cos \left(\theta_{\mathrm{RX}, k}\right), \sin \left(\theta_{\mathrm{RX}, k}\right), 0\right]^{\mathrm{T}}, \\
& \mathbf{v}_{A, k}^{(\mathrm{G})}=v_{A, k}^{(\mathrm{G})}\left[-\sin \left(\theta_{\mathrm{RX}, k}\right), \cos \left(\theta_{\mathrm{RX}, k}\right),\left\|\mathbf{p}-\mathbf{s}_{k}\right\|\right]^{\mathrm{T}},
\end{aligned}
$$

respectively. The term $v_{A, k}^{(\mathrm{G})}=\sqrt{1 /\left(1+\left\|\mathbf{p}-\mathbf{s}_{k}\right\|^{2}\right)}$ normalizes the eigenvector.

Interpretation: AOA and TOA of each NLOS component contribute to the EFIM. The eigenvalues can be interpreted as the achievable information gains if the points of incidence were perfectly known. From (35a), we see that TOA provides only position information. From (35b), we deduce that AOA provides both position and orientation information. The term in (34b) suggests that the information gain of NLOS components decreases as the separation between the mobile terminal and point of incidence increases. For large distances, the information gain converges to the inverse of the AOA estimation accuracy $1 / \sigma_{\mathrm{RX}, k}^{2}$. Observe that in this case, a NLOS component provides mostly orientation information, since the $x-y$ components of the eigenvector in (35b) go to zero. Note that AOD estimation does not provide any information gain. However, we will see in the next section that the quality of the AOD estimation strongly influences the information loss.

\section{NLOS Information Loss}

The NLOS information loss matrix is not as obvious to decompose as the previous decompositions since it involves multiple matrix-matrix products including the inverse of a matrix-matrix product. In the following, we will present the results of the decomposition.

Lemma 4. The NLOS information loss matrix is given by

$$
\begin{aligned}
\tilde{\mathbf{B}}^{(\mathrm{L})}=\sum_{k=1}^{K-1} & \underbrace{w_{R, k}^{(\mathrm{L})} \boldsymbol{\Upsilon}_{0,0}\left(\theta_{\mathrm{TX}, 0}, 0,0\right)}_{\tilde{\mathbf{B}}_{R, k}^{(\mathrm{L})}} \\
& +\underbrace{w_{A, k}^{(\mathrm{L})} \mathbf{\Upsilon}_{1,1}\left(\theta_{\mathrm{RX}, k}, \pi / 2,\left\|\mathbf{p}-\mathbf{s}_{k}\right\|\right)}_{\tilde{\mathbf{B}}_{A, k}^{(\mathrm{L})}} \\
& -\gamma_{\mathbf{s}_{k}} \mathbf{B}_{k}^{(\mathrm{L})},
\end{aligned}
$$

where the weights $9 w_{R, k}^{(\mathrm{L})}, w_{A, k}^{(\mathrm{L})}$ and $\gamma_{\mathbf{s}_{k}}$, and the matrix $\mathbf{B}_{k}^{(\mathrm{L})}$ are defined in Appendix $C$ - $B$.

Proof. See Appendix C-B.

\footnotetext{
${ }^{9}$ Each weight is a function of $\left(\theta_{\mathrm{TX}, k}, \theta_{\mathrm{RX}, k}, \tau_{k}, \sigma_{\theta_{\mathrm{TX}, k}}^{2}, \sigma_{\theta_{\mathrm{RX}, k}}^{2}, \sigma_{\tau_{k}}^{2}, \mathbf{p}\right.$
} $\left.\mathbf{q}, \mathbf{s}_{k}\right)$. To simplify notation, we dropped the arguments. 


$$
\lambda_{\mathbf{s}_{k}} \triangleq \frac{2+\left\|\mathbf{p}-\mathbf{s}_{k}\right\|^{2}\left(1+\cos \left(\Delta \theta_{k}\right)\right)}{\left(1-\cos \left(\Delta \theta_{k}\right)\right) c^{2} \sigma_{\tau_{k}}^{2}+\left(1+\cos \left(\Delta \theta_{k}\right)\right)\left(\left\|\mathbf{p}-\mathbf{s}_{k}\right\|^{2} \sigma_{\theta_{\mathrm{RX}, k}}^{2}+\left\|\mathbf{q}-\mathbf{s}_{k}\right\|^{2} \sigma_{\theta_{\mathrm{TX}, k}}^{2}\right)}
$$

Interpretation: The information loss of each path is composed of three terms. Comparing (33) and (36), we see that the first two terms of every path $k$ have the same structure. In particular, the TOA information gain $\tilde{\mathbf{B}}_{R, k}^{(\mathrm{G})}$ and loss $\tilde{\mathbf{B}}_{R, k}^{(\mathrm{L})}$ only differ in the weights. The same holds for the AOA information gain $\tilde{\mathbf{B}}_{A, k}^{(\mathrm{G})}$ and loss $\tilde{\mathbf{B}}_{A, k}^{(\mathrm{L})}$. Hence each information gain and loss pair has eigenvectors that are pointing in the same direction. Yet, the eigenvalues have opposite signs. For these terms, net information gains are immediately given by the differences of the respective weights. On the other hand, the third term has eigenvectors which are not aligned with any other eigenvectors. In the following subsection, we will show that combining the gain and loss matrices of every NLOS component results in a net information gain matrix which has only a single non-zero, positive eigenvalue.

\section{E. Net NLOS Information Gain}

Lemma 5. The net NLOS gain matrix $\tilde{\mathbf{B}}^{(\mathrm{N})} \triangleq \tilde{\mathbf{B}}^{(\mathrm{G})}-\tilde{\mathbf{B}}^{(\mathrm{L})}$ is given by

$$
\begin{aligned}
\tilde{\mathbf{B}}^{(\mathrm{N})}=\sum_{k=1}^{K-1} & \epsilon_{\mathbf{s}_{k}} \boldsymbol{\Upsilon}_{0,0}\left(\theta_{\mathrm{RX}, 0}, 0,0\right) \\
& +\beta_{\mathbf{s}_{k}} \mathbf{\Upsilon}_{1,1}\left(\theta_{\mathrm{RX}, k}, \pi / 2,\|\mathbf{p}-\mathbf{q}\|\right) \\
& +\gamma_{\mathbf{s}_{k}} \mathbf{B}_{k}^{(\mathrm{L})}
\end{aligned}
$$

where $\epsilon_{\mathbf{s}_{k}} \triangleq \frac{1}{\sigma_{\tau_{k}}^{2} c^{2}}-w_{R, k}^{(\mathrm{L})}$ and $\beta_{\mathbf{s}_{k}} \triangleq \frac{1}{\sigma_{\theta_{\mathrm{RX}, k}}^{2}\left\|\mathbf{p}-\mathbf{s}_{k}\right\|^{2}}-w_{A, k}^{(\mathrm{L})}$.

Proof. Applying Lemma 3 and 4, we immediately obtain (38).

We define $\tilde{\mathbf{B}}^{(N)} \triangleq \sum_{k=1}^{K-1} \mathbf{\Psi}_{\mathbf{s}_{k}}$, where

$$
\begin{aligned}
\boldsymbol{\Psi}_{\mathbf{s}_{k}} \triangleq & \epsilon_{\mathbf{s}_{k}} \boldsymbol{\Upsilon}_{0,0}\left(\theta_{\mathrm{TX}, 0}, 0,0\right)+ \\
& \beta_{\mathbf{s}_{k}} \boldsymbol{\Upsilon}_{1,1}\left(\theta_{\mathrm{RX}, k}, \pi / 2,\|\mathbf{p}-\mathbf{q}\|\right)+\gamma_{\mathbf{s}_{k}} \mathbf{B}_{k}^{(\mathrm{L})} .
\end{aligned}
$$

Theorem 1. The net information gain matrix $\boldsymbol{\Psi}_{\mathbf{s}_{k}}$ of the $k^{\text {th }}$ NLOS component is rank one. The only non-zero eigenvalue of $\boldsymbol{\Psi}_{\mathbf{s}_{k}}$ is always positive and given by (37) at the top of this page with the corresponding unit-norm eigenvector

$$
\mathbf{v}_{\mathbf{s}_{k}} \triangleq v_{\mathbf{s}_{k}}\left[\begin{array}{c}
-\frac{\epsilon_{\mathbf{s}_{k}}}{\gamma_{\mathbf{s}_{k}}} \cos \left(\theta_{\mathrm{RX}, k}\right)-\sin \left(\theta_{\mathrm{RX}, k}\right) \\
-\frac{\epsilon_{\mathbf{s}_{k}}}{\gamma_{\mathbf{s}_{k}}} \sin \left(\theta_{\mathrm{RX}, k}\right)+\cos \left(\theta_{\mathrm{RX}, k}\right) \\
\left\|\mathbf{p}-\mathbf{s}_{k}\right\|
\end{array}\right]
$$

where $\Delta \theta_{k} \triangleq \theta_{\mathrm{RX}, k}-\theta_{\mathrm{TX}, k} \quad$ and $^{10}$
$\sqrt{\left(1+\cos \left(\Delta \theta_{k}\right)\right) /\left(2+\left\|\mathbf{p}-\mathbf{s}_{k}\right\|^{2}\left(1+\cos \left(\Delta \theta_{k}\right)\right)\right)}$

Proof. Outline: using Lemma 5, it can be shown that $\boldsymbol{\Psi}_{\mathbf{s}_{k}}$ is rank one. Hence the only non-zero eigenvalue is given by $\lambda_{\mathbf{s}_{k}}=\operatorname{tr}\left(\boldsymbol{\Psi}_{\mathbf{s}_{k}}\right)$. Positivity of $\lambda_{\mathbf{s}_{k}}$ is readily seen since all

\footnotetext{
${ }^{10}$ The normalization constant $v_{\mathbf{s}_{k}}$ can be easily verified considering $\frac{\epsilon_{\mathbf{s}_{k}}}{\gamma_{\mathbf{s}_{k}}}=$ $\frac{1-\cos \left(\Delta \theta_{k}\right)}{\sin \left(\Delta \theta_{k}\right)}$
}

terms in (37) are non-negative, i.e. $\left(1+\cos \left(\theta_{\mathrm{RX}, k}-\theta_{\mathrm{TX}, k}\right)\right) \geq$ $0,\left(1-\cos \left(\theta_{\mathrm{RX}, k}-\theta_{\mathrm{TX}, k}\right)\right) \geq 0$, and all other terms are positive by definition. Finally, we show that the vector $\mathbf{v}_{\mathbf{s}_{k}}$ in (40) is in the null space of $\left(\lambda_{\mathbf{s}_{k}} \mathbf{I}-\mathbf{\Psi}_{\mathbf{s}_{k}}\right)$. Hence $\mathbf{v}_{\mathbf{s}_{k}}$ is the corresponding eigenvector. The details can be found in Appendix C-C.

Interpretation: Each NLOS component provides one dimensional Fisher information for all three parameters $\left(p_{x}\right.$, $p_{y}$, and $\alpha$ ). The information of all NLOS components is additive and, thus, contributes to the EFIM which, in turn, reduces the position and orientation error bound. Hence NLOS components can be harnessed to increase the position and orientation estimation accuracy in 5G mmWave MIMO systems. The amount of information gained from a NLOS component depends strongly on the geometry (this can be seen from the $\cos \left(\Delta \theta_{k}\right)$-terms in (37)), i.e. points of incidence which are located in certain areas provide more information than points of incidence in other areas. From the denominator in (37), it can be seen that NLOS components provide considerable Fisher information if and only if TOA, AOD, and AOA can be estimated sufficiently accurate. If any of the three quantities cannot be estimated, e.g., AOD, this is equivalent to $\sigma_{\theta_{\mathrm{TX}, k}}^{2} \rightarrow \infty$. Hence $\lambda_{\mathbf{s}_{k}} \rightarrow 0$ which means that the NLOS component does not provide useful information for position and orientation estimation. Finally, we see that points of incidence which are close to the base station or the mobile terminal (i.e. with small $\left\|\mathbf{q}-\mathbf{s}_{k}\right\|$ or $\left\|\mathbf{p}-\mathbf{s}_{k}\right\|$, respectively) are more informative than points of incidence which are farther away. Intuitively, this observation makes sense because, for a given angular estimation accuracy, the position of a point of incidence can be estimated better if it is close the base station. If it is far away from the base station, a small angular estimation error translates into a large estimation error of the point of incidence. The same holds for the path from the point of incidence to the mobile terminal.

Corollary 1.1. The EFIM of position and orientation is given by the sum of the outer-products of the eigenvectors weighted by the corresponding eigenvalues, i.e.,

$$
\mathbf{J}_{\check{\eta}_{\mathbf{p}, \alpha}}^{\mathrm{e}}=\sum_{j \in R, D, A} \lambda_{j, 0}^{(\mathrm{G})} \mathbf{v}_{j, 0}^{(\mathrm{G})}\left(\mathbf{v}_{j, 0}^{(\mathrm{G})}\right)^{\mathrm{T}}+\sum_{k=1}^{K-1} \lambda_{\mathbf{s}_{k}} \mathbf{v}_{\mathbf{s}_{k}} \mathbf{v}_{\mathbf{s}_{k}}^{\mathrm{T}} .
$$

Proof. Considering Lemma 1 and Theorem 1, we know that each matrix in (27) is rank one. Since any rank one matrix can be written as the outer-product of the unit-norm eigenvector with itself and weighted by the only non-zero eigenvalue, (41) follows.

Interpretation: Note that the position and orientation of the receiver can be determined if $\mathbf{J}_{\grave{\eta}_{\mathbf{p}, \alpha}}^{\mathrm{e}}$ is non-singular. From (41), we observe that even in the absence of the LOS component, unambiguous position and orientation estimation is possible 
if at least three NLOS components with distinct points of incidence contribute to the received signal.

For illustration purposes, we consider the position components and the orientation component of the eigenvalueeigenvector product $\lambda_{\mathbf{s}_{k}} \mathbf{v}_{\mathbf{s}_{k}}$ separately. This can be interpreted as the projection of the eigenvalue-eigenvector product on the $\mathrm{x}-\mathrm{y}$ plane (position components) and on the $\alpha$ line (orientation component), respectively. After a few algebraic manipulations, it can be shown that the length of the projected eigenvalueeigenvector product in the $\mathrm{x}-\mathrm{y}$ plane is given by

$$
\tilde{\lambda}_{\mathbf{s}_{k}} \triangleq \lambda_{\mathbf{s}_{k}} \sqrt{v_{\mathbf{s}_{k}, x}^{2}+v_{\mathbf{s}_{k}, y}^{2}}
$$

where $v_{\mathbf{s}_{k}, x}$ and $v_{\mathbf{s}_{k}, y}$ denote the first and second component of $\mathbf{v}_{\mathbf{s}_{k}}$, respectively. The length of the projected eigenvalueeigenvector product along the $\alpha$ line is given by

$$
\bar{\lambda}_{\mathbf{s}_{k}} \triangleq \lambda_{\mathbf{s}_{k}} v_{\mathbf{s}_{k}, \alpha}
$$

where $v_{\mathbf{s}_{k}, \alpha}$ denotes the third component of $\mathbf{v}_{\mathbf{s}_{k}}$. Finally, the position error bound is defined as

$$
\mathrm{PEB}=\sqrt{\operatorname{tr}\left\{\left[\left(\mathbf{J}_{\breve{\eta}_{\mathbf{p}, \alpha}}^{\mathrm{e}}\right)^{-1}\right]_{1: 2,1: 2}\right\}} .
$$

\section{F. Discussion and Implications}

Our findings provide insights into the problem of position and orientation estimation in $5 \mathrm{G}$ mmWave MIMO. Our results may turn out to be useful for designing position and orientation estimators. For instance, Theorem 1 revealed that every NLOS path provides position and orientation information, and hence every NLOS path reduces the PEB. To maximize the estimation accuracy, a position and orientation estimator should be designed such that all NLOS paths are considered. On the other hand, we know from Theorem 1 that the net NLOS information gain among paths can vary strongly, i.e. some NLOS paths provide significantly more information than others. To reduce the complexity of an estimator, paths with insignificant net NLOS information gain could be neglected by an estimator without considerable losses in terms of accuracy. For a given observation, the problem whether to consider or neglect a NLOS path is still an open challenge, which requires further research.

Theorem 1 inherently implies that the position of the point of incidence of a NLOS path can be estimated. Thus, simultaneous localization and mapping (SLAM) can be performed with single transmission burst. Moreover, our results expose some explicit laws, e.g., (40) shows that a NLOS path, whose point of incidence is far away from the mobile terminal, contains mainly orientation information and only marginal position information. From Corollary 1.1, it can be seen that in the absence of the LOS path, at least three NLOS paths lead to a full rank EFIM. Hence NLOS-only position and orientation estimation are possible with solely a single anchor if at least three NLOS are available.

\section{NuMERICAL EXAMPLE}

In this section, we provide numerical examples for position and orientation estimation using mmWave MIMO with typical $5 \mathrm{G}$ parameters. First, we describe the simulation setup. Subsequently, we present two examples which 1) discuss the reduction of the PEB when an NLOS path is added to the LOS path and 2) demonstrate LOS-free, single-anchor localization.

\section{A. Simulation Setup}

We consider a mobile terminal that is located at $\mathbf{p}=[5,5]^{\mathrm{T}}$ with an orientation angle of $\alpha=\pi / 2$, while the base station in located at $\mathbf{q}=[0,0]^{\mathrm{T}}$. In contrast to [20], we consider uniform linear arrays (ULAs) with half-wavelength inter-element spacing at the transmitter and receiver that consist of $N_{\mathrm{TX}}$ and $N_{\mathrm{RX}}=25$ antennas, respectively. The operating carrier frequency is $f_{c}=38 \mathrm{GHz}$. Regarding the pilot signal, we consider an ideal sinc pulse with $B=125 \mathrm{MHz}, E_{\mathrm{s}} / T_{\mathrm{s}}=0 \mathrm{dBm}$, $N_{0}=-170 \mathrm{dBm} / \mathrm{Hz}$, and $N_{\mathrm{s}}=16$ symbols. We assume that $N_{B}=50$ beams are transmitted which are uniformly spaced in the azimuth domain. In particular, the beamforming vectors ${ }^{11}$ are given by $\mathbf{f}_{l}\left(\theta_{\mathrm{BF}, l}\right)=1 / \sqrt{N_{\mathrm{B}}} \mathbf{a}_{\mathrm{TX}}\left(\theta_{\mathrm{BF}, l}\right)$, where $\theta_{\mathrm{BF}, l}$ is the $l^{\text {th }}$ element of the vector $\boldsymbol{\theta}_{\mathrm{BF}}=\pi / 50 \cdot[1, \ldots, 50]^{T}$. The complex channel gains for each path are generated according to a geometric model [43]. The gain is proportional to the path loss and the phase is uniformly distributed. In particular, we assume that $\left|h_{0}\right|^{2}=(\lambda / 4 \pi)^{2} /\|\mathbf{p}-\mathbf{q}\|^{2}$ for the LOS path and $\left|h_{k}\right|^{2}=(\lambda / 4 \pi)^{2} \Gamma_{R} /\left(\left\|\mathbf{q}-\mathbf{s}_{k}\right\|+\left\|\mathbf{p}-\mathbf{s}_{k}\right\|\right)^{2}, k>0$ for NLOS paths ${ }^{12}$, where $\Gamma_{R}=0.7$. The theoretical results presented in the previous sections also hold for other sets of parameters as long as the FIM of the channel parameters is almost diagonal [20].

\section{B. Example 1}

We consider the LOS path and one NLOS path in this example. The reflector, which causes the NLOS path, is moved in the $\mathrm{x}-\mathrm{y}$ plane between $0<s_{\mathrm{x}, 1} \leq 10$ and $0<s_{\mathrm{y}, 1} \leq 10$. For every location $\mathbf{s}_{1}$ of the point of reflection, we determine the net position information gain $\tilde{\lambda}_{\mathbf{s}_{1}}$, which is depicted in logscale in Fig. 2. The array of the mobile terminal is shown in black. We consider different transmit array sizes to highlight the effect of the beamwidth on the position information gain. In particular, we choose $N_{\mathrm{TX}}=25$ (Fig. 2 - left) and $N_{\mathrm{TX}}=150$ (Fig. 2 - right). Wider beams of the former array result in a homogeneous illumination of the plane, which makes it more obvious to point out the location dependency of the point of incidence on the net position information gain. Three main conclusions can be drawn:

1) First, the geometry of the scenario has a significant impact on the net position information gain. The results

\footnotetext{
${ }^{11}$ We stress that our study can be applied to any kind of precoding. If hybrid transceiver and receiver architectures are considered, the flexibility of the beams that can be generated decreases compared to fully digital architectures, leading to lower positioning accuracy [42]. In general, it can be observed that wide beams result in lower position estimation accuracy compared to narrow beams, as will be shown in Fig. 3 .

${ }^{12}$ For the numerical examples, we consider the point of incidence of a reflector as the source of the NLOS path.
} 

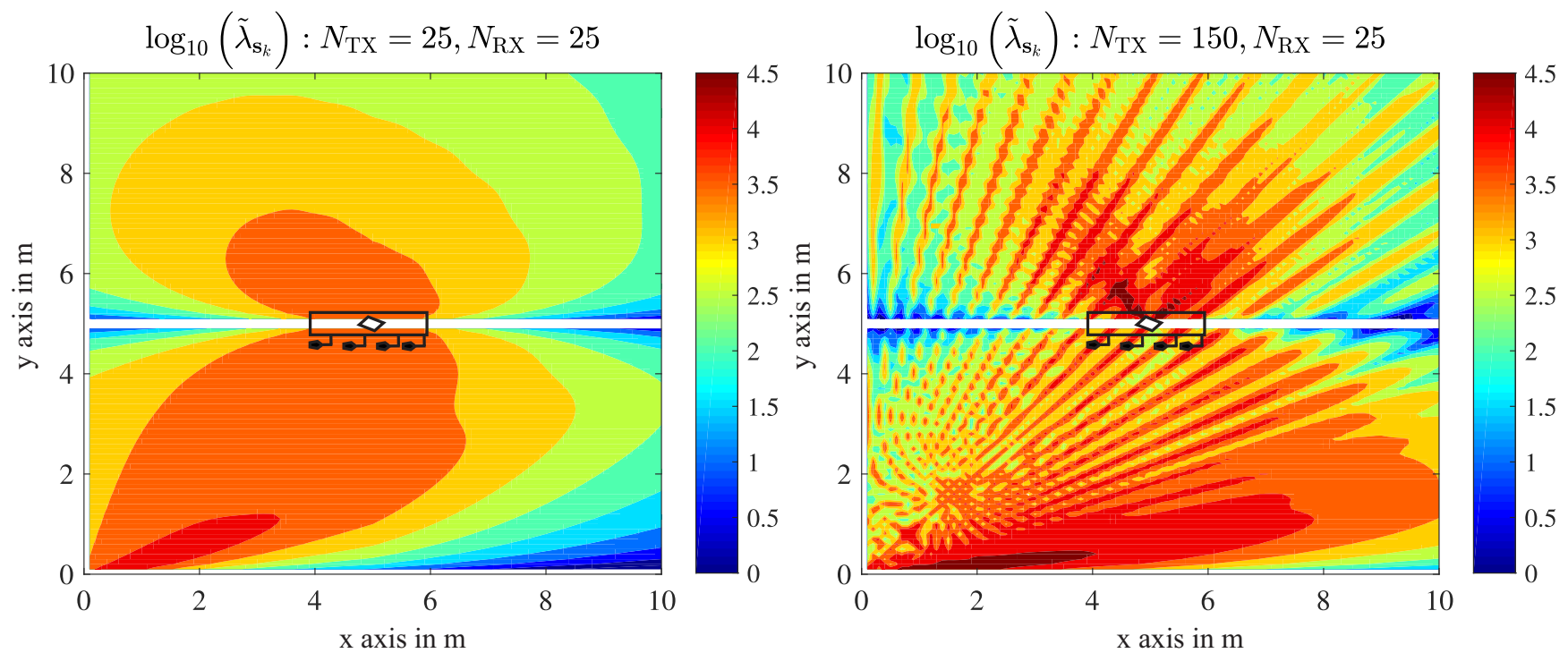

Fig. 2: Net position information gain - Narrower beamwidth caused by more transmit antennas (right) allows for larger net position information gain when compared to wider beamwidth (left).
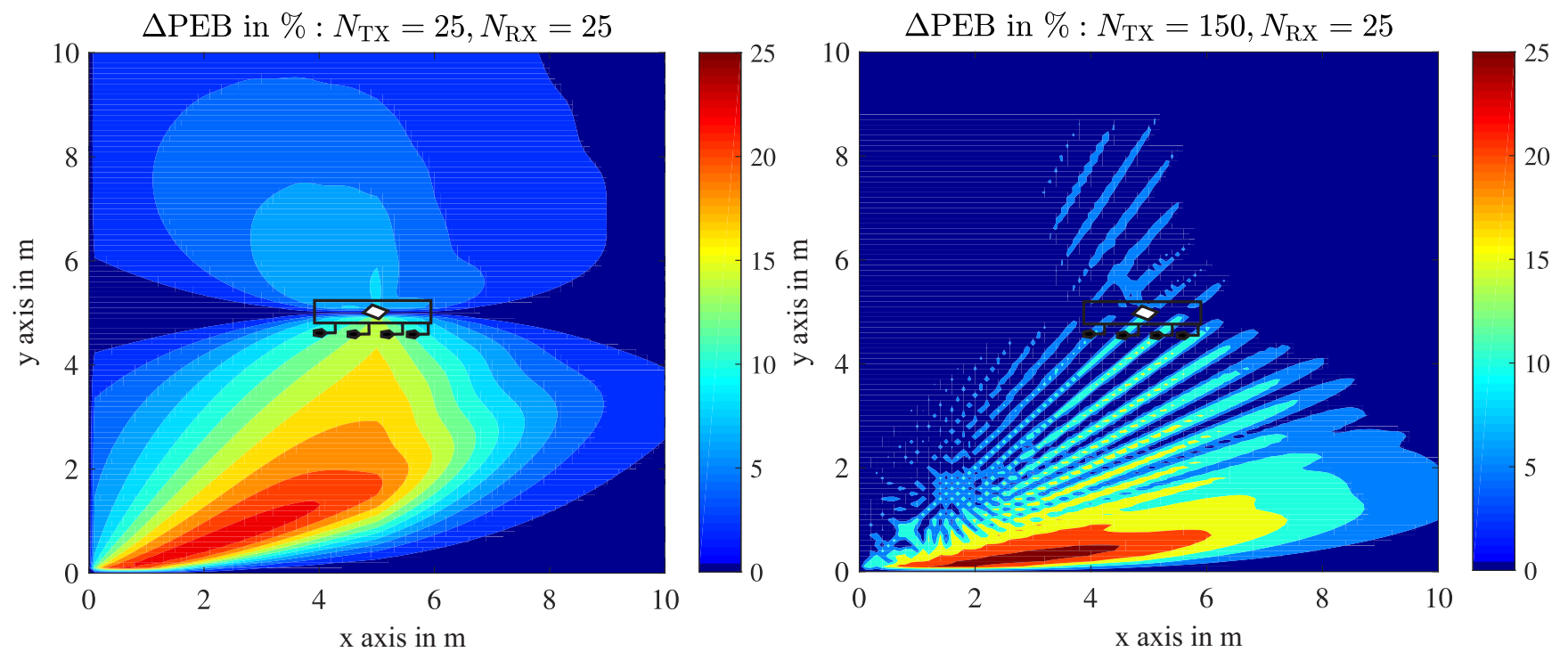

Fig. 3: Reduction of the PEB - The patterns of the reductions of the PEBs closely resemble the patterns of the net position information gains in Fig. 2.

in Fig. 2 (left) confirm our findings from the analysis on the position information gain in section IV-E. In particular, points of incidence that are close to the transmitter and receiver provide large information gains. One can observe in (42) that the net position gain is a function of $\Delta \theta_{k}, \mathbf{q}, \mathbf{p}$, and $\mathbf{s}_{k}$. Hence the net position information gain is a function of the geometry which implies that certain NLOS paths result in larger net position information than others. This can be deduced from the inhomogeneous color pattern in Fig. 2.

2) Secondly, the illumination of the plane with the transmitted beams has a major impact on the position information gain of NLOS components. Generally, narrower beams generated by larger apertures result in larger net position information gains. Thus, the points of incidence of NLOS paths should be illuminated with beams which are as narrow as possible, in order to obtain the highest increase in the positioning accuracy.

3) The fact that NLOS components provide information regarding the position of the mobile terminal implies that the points of incidence of NLOS paths themselves can be estimated. Hence simultaneous localization and mapping (SLAM) can be conducted with a single snapshot. Thus, if SLAM is the goal on the system level, denselyspaced narrow beams are preferable over wide beams in order to accurately estimate the positions of the reflectors and achieve high localization accuracy of the mobile terminal. A synergy between communication and environmental mapping can be identified. When highly accurate maps of the environment have been obtained, e.g., in the initial access phase, they can be stored in a cloud database. Such a cloud database can, in turn, 

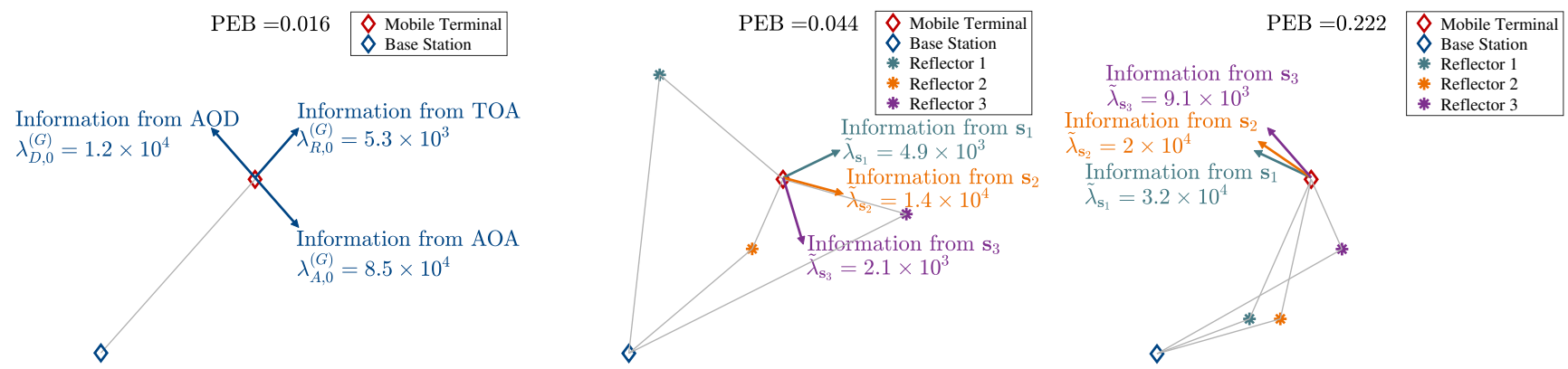

Fig. 4: Position information gain and direction - Left: position information gains and directions originating from TOA, AOD, and AOA of the LOS path. Middle and right: net position information gains and directions of 3 NLOS paths. Accurate positioning is possible even in the absence of the LOS path but positioning accuracy degrades as the reflectors become densely-spaced in the AOD-domain.

be used to support communication, since beams could be intentionally steered towards points of incidence to increase the capacity or reliably of the link.

Even though, we did not analytically show the reduction of the PEB in the presence of a NLOS path, we provide a numerical analysis. Note that the increased net position information gain does not necessarily lead to a reduction of the PEB since determining the PEB requires to take the trace of the inverse of the EFIM. The EFIM also depends on the outer products of the eigenvectors of the NLOS paths $\lambda_{\mathbf{s}_{k}} \mathbf{v}_{\mathbf{s}_{k}} \mathbf{v}_{\mathbf{s}_{k}}^{\mathrm{T}}$ and the outer products of the eigenvectors of the LOS path $\lambda_{j, 0}^{(\mathrm{G})} \mathbf{v}_{j, 0}^{(\mathrm{G})}\left(\mathbf{v}_{j, 0}^{(\mathrm{G})}\right)^{\mathrm{T}}, j \in\{R, D, A\}$. Nonetheless, the Figs. 2 and 3 suggest that in many cases a large net position information gain also results in a large reduction of the PEB. We determine the reduction of the position error bound $\triangle \mathrm{PEB}$ numerically in the presence of a single NLOS paths and depict a heat map in Fig. 3. We observe that the presence of the reflector reduces the PEB by up to $25 \%$. Narrower beams (Fig. 3 right) result in larger reductions of the PEB. Note that the patterns in Fig. 3 closely resemble the patterns of the net position information gain in Fig. 2, i.e. when the net position information gain is large, the reduction of the PEB is also large. It has been observed by OEB simulations (not shown), that the relationships governing orientation information gain and the reduction of the OEB are similar to those related with the net position information gain and the reduction of the PEB, respectively. Thus, for the sake of brevity, no results regarding the orientation are presented.

\section{Example 2}

We assume that the transmitter and the receiver are equipped with $N_{\mathrm{TX}}=N_{\mathrm{TX}}=25$ antennas, and the plane is illuminated with $N_{\mathrm{B}}=50$ beams. We consider three different cases which are depicted in Fig. 4:

1) a single LOS path

2) three NLOS paths with reflectors distributed uniformly around the mobile terminal (reflectors are located at $\mathbf{s}_{1}=[1,8]^{\mathrm{T}}, \mathbf{s}_{2}=[4,3]^{\mathrm{T}}$, and $\left.\mathbf{s}_{3}=[8,4]^{\mathrm{T}}\right)$

3) three NLOS paths with reflectors that are denselyspaced in the AOD-domain (reflectors are located at $\mathbf{s}_{1}=[3,1]^{\mathrm{T}}, \mathbf{s}_{2}=[4,1]^{\mathrm{T}}$, and $\left.\mathbf{s}_{3}=[6,3]^{\mathrm{T}}\right)$
Case 2) emulates an initial access scenario, where the entire plane is illuminated and reflectors may be located far apart from each other, while case 3) takes into consideration that reflectors tend to appear densely-spaced in the AOD-domain. The underlying assumption behind case 3 ) is that during data transmission only a few narrow beams are used and reflectors which are not in the vicinity of the main lobe of these beams will not be illuminated.

In Fig. 4, the direction of information (eigenvector) is indicated by normalized colored arrows, while the net position information gain (eigenvalue) is presented in text boxes attached to the arrows. The corresponding PEB is provided at the top of the respective sub-figure. The following conclusions can be drawn for the respective scenarios:

1) We can deduce from Fig. 4 (left) that unambiguous single-anchor position and orientation estimation is possible. We obtain $\mathrm{PEB}_{1}=0.016$.

2) From Fig. 4 (middle), we can infer that every NLOS path originating from a reflectors has a different direction of information, leading to a full rank EFIM as discussed in Corollary 1.1. Compared to case 1) (LOS), the PEB is approximately 3 times higher $\left(\mathrm{PEB}_{2}=0.044 \approx 3\right.$. $\mathrm{PEB}_{1}$ ). This result suggest that even in the absence of the LOS path, the position estimation accuracy will only be slightly impaired (compared to the LOS case) if the reflectors are geometrically well distributed.

3) Fig. 4 (right) depicts case 3), where the reflectors are densely-spaced in the AOD-domain. We can infer that if the reflectors are densely-spaced in the AOD-domain, the direction of information will also be correlated. In that case, the eigenvectors of the rank-one EFIMs in (41) are fairly similar, as can be seen in Fig. 4 (right). In that case, unambiguous positioning is still possible but the PEB is significantly higher, i.e. $\mathrm{PEB}_{3}=0.222 \gg$ $\mathrm{PEB}_{2}$.

\section{CONCLUSION}

We analyzed the role of NLOS components in 5G mmWave MIMO systems in terms of their position and orientation estimation capabilities. For our analysis, we employed the concept of Fisher information in order to show that NLOS components provide significant Fisher information if and 


$$
\begin{gathered}
\mathbf{T}_{\mathbf{P}}^{(0)}=\left[\begin{array}{ccc}
\frac{1}{c} \cos \left(\theta_{\mathrm{TX}, 0}\right) & -\frac{1}{\|\mathbf{p}-\mathbf{q}\|} \sin \left(\theta_{\mathrm{TX}, 0}\right) & -\frac{1}{\|\mathbf{p}-\mathbf{q}\|} \sin \left(\theta_{\mathrm{TX}, 0}\right) \\
\frac{1}{c} \sin \left(\theta_{\mathrm{TX}, 0}\right) & \frac{1}{\|\mathbf{p}-\mathbf{q}\|} \cos \left(\theta_{\mathrm{TX}, 0}\right) & \frac{1}{\|\mathbf{p}-\mathbf{q}\|} \cos \left(\theta_{\mathrm{TX}, 0}\right) \\
0 & 0 & -1
\end{array}\right] \\
\mathbf{T}_{\mathbf{P}}^{(k)}=\left[\begin{array}{ccc}
\frac{1}{c} \cos \left(\pi-\theta_{\mathrm{RX}, k}\right) & 0 & -\frac{1}{\| \mathbf{p}^{-\mathbf{s}_{k} \|}} \sin \left(\pi-\theta_{\mathrm{RX}, k}\right) \\
-\frac{1}{c} \sin \left(\pi-\theta_{\mathrm{RX}, k}\right) & 0 & \frac{1}{\left\|\mathbf{p}-\mathbf{s}_{k}\right\|} \cos \left(\pi-\theta_{\mathrm{RX}, k}\right) \\
0 & 0 & -1
\end{array}\right] \\
\mathbf{T}_{\mathbf{s}_{k}}=\left[\begin{array}{ccc}
\frac{1}{c}\left[\cos \left(\theta_{\mathrm{TX}, k}\right)+\cos \left(\theta_{\mathrm{RX}, k}\right)\right] & -\frac{1}{\left\|\mathbf{q}-\mathbf{s}_{k}\right\|} \sin \left(\theta_{\mathrm{TX}, k}\right) & -\frac{1}{\left\|\mathbf{p}-\mathbf{s}_{k}\right\|} \sin \left(\pi-\theta_{\mathrm{RX}, k}\right) \\
\frac{1}{c}\left[\sin \left(\theta_{\mathrm{TX}, k}\right)+\sin \left(\theta_{\mathrm{RX}, k}\right)\right] & \frac{1}{\left\|\mathbf{q}-\mathbf{s}_{k}\right\|} \cos \left(\theta_{\mathrm{TX}, k}\right) & -\frac{1}{\left\|\mathbf{p}-\mathbf{s}_{k}\right\|} \cos \left(\pi \theta_{\mathrm{RX}, k}\right)
\end{array}\right]
\end{gathered}
$$

only if angle-of-arrival, angle-of-departure, and time-of-arrival of the corresponding path can be estimated accurately. We showed analytically that each NLOS component contributes one dimensional Fisher information regarding the position and orientation. Hence NLOS components can be harnessed to increase position and orientation accuracy. We showed that even in the absence of the LOS component, unambiguous position and orientation estimation is feasible if at least three NLOS paths contribute to the received signal. We showed in our analysis that the amount of gained information strongly depends on the relative position of the mobile terminal, the base station, and the points of incidence, as well as the illumination of the point of incidence by base station. We pointed out that, for a given received SNR, narrow beams increase the position and orientation information gain of NLOS components when compared to wider beams.

\section{APPENDIX A}

\section{ENTRIES OF THE TRANSFORMATION MATRIX}

The entries of the block-matrices in $\mathbf{T}$ are given by (45)(47) [19] at the top of this page.

\section{APPENDIX B}

\section{EIGENVALUES AND EIGENVECTORS OF THE LOS INFORMATION GAIN MATRICES}

Since the matrices in (30) are rank one, the eigenvalues are given by the traces of the respective matrices [44]. Hence (31) follow.

Having obtained the eigenvalues of the matrices $\tilde{\mathbf{A}}_{R}^{(\mathrm{G})}, \tilde{\mathbf{A}}_{D}^{(\mathrm{G})}$, and $\tilde{\mathbf{A}}_{A}^{(\mathrm{G})}$, it is straightforward to see that the vectors $\mathbf{v}_{R, 0}^{(\mathrm{G})}$, $\mathbf{v}_{D, 0}^{(\mathrm{G})}$, and $\mathbf{v}_{A, 0}^{(\mathrm{G})}$ in (32) are in the null space of $\left(\lambda_{R}^{(\mathrm{G})} \mathbf{I}-\tilde{\mathbf{A}}_{R}^{(\mathrm{G})}\right)$, $\left(\lambda_{D}^{(\mathrm{G})} \mathbf{I}-\tilde{\mathbf{A}}_{D}^{(\mathrm{G})}\right)$, and $\left(\lambda_{A}^{(\mathrm{G})} \mathbf{I}-\tilde{\mathbf{A}}_{A}^{(\mathrm{G})}\right)$, respectively, i.e.

$$
\begin{aligned}
& \left(\lambda_{R}^{(\mathrm{G})} \mathbf{I}-\tilde{\mathbf{A}}_{R}^{(\mathrm{G})}\right) \mathbf{v}_{R, 0}^{(\mathrm{G})}=\mathbf{0}, \\
& \left(\lambda_{D}^{(\mathrm{G})} \mathbf{I}-\tilde{\mathbf{A}}_{D}^{(\mathrm{G})}\right) \mathbf{v}_{D, 0}^{(\mathrm{G})}=\mathbf{0}, \\
& \left(\lambda_{A}^{(\mathrm{G})} \mathbf{I}-\tilde{\mathbf{A}}_{A}^{(\mathrm{G})}\right) \mathbf{v}_{A, 0}^{(\mathrm{G})}=\mathbf{0} .
\end{aligned}
$$

\section{APPENDIX C}

\section{DECOMPOSITION OF THE NLOS INFORMATION LOSS}

In the following, we will derive the decomposition of the terms $\tilde{\mathbf{B}}^{(\mathrm{G})}, \tilde{\mathbf{B}}^{(\mathrm{L})}$, and $\tilde{\mathbf{B}}^{(\mathrm{N})}=\tilde{\mathbf{B}}^{(\mathrm{G})}-\tilde{\mathbf{B}}^{(\mathrm{L})}$.

\section{A. NLOS Information Gain}

The NLOS information gain is given by

$$
\begin{aligned}
\tilde{\mathbf{B}}^{(\mathrm{G}) \triangleq} & \mathbf{B} \mathbf{J}_{\overline{\boldsymbol{\eta}}_{\mathrm{NLOS}}} \mathbf{B}^{\mathrm{T}} \\
= & {\left[\mathbf{T}_{\mathbf{P}}^{(1)}, \mathbf{T}_{\mathbf{P}}^{(2)}, \ldots, \mathbf{T}_{\mathbf{P}}^{(K-1)}\right] } \\
& \times \mathbf{J}_{\overline{\boldsymbol{\eta}}_{\mathrm{NLOS}}}\left[\mathbf{T}_{\mathbf{P}}^{(1)}, \mathbf{T}_{\mathbf{P}}^{(2)}, \ldots, \mathbf{T}_{\mathbf{P}}^{(K-1)}\right]^{\mathrm{T}} .
\end{aligned}
$$

We find

$$
\begin{aligned}
\tilde{\mathbf{B}}^{(\mathrm{G})} & =\sum_{k=1}^{K-1} \mathbf{T}_{\mathbf{P}}^{(k)} \mathbf{J}_{\overline{\boldsymbol{\eta}}_{k}}\left(\mathbf{T}_{\mathbf{P}}^{(k)}\right)^{\mathrm{T}} \\
& =\sum_{k=1}^{K-1} \frac{1}{\sigma_{\tau_{k}}^{2} c^{2}} \mathbf{\Upsilon}_{0,0}\left(\theta_{\mathrm{RX}, k}, 0,0\right) \\
& +\frac{1}{\sigma_{\theta_{\mathrm{RX}, k}}^{2}\left\|\mathbf{p}-\mathbf{s}_{k}\right\|^{2}} \mathbf{\Upsilon}_{1,1}\left(\theta_{\mathrm{RX}, k}, \pi / 2,\left\|\mathbf{p}-\mathbf{s}_{k}\right\|\right)
\end{aligned}
$$

\section{B. NLOS Information Loss}

It is easy to verify that $\mathbf{D} \mathbf{J}_{\overline{\boldsymbol{\eta}}_{\mathrm{NLOS}}} \mathbf{D}^{\mathrm{T}}$ is block diagonal since $\mathbf{D}$ and $\mathbf{J}_{\overline{\boldsymbol{\eta}}_{\mathrm{NLOS}}}$ are block diagonal, i.e.

$$
\mathbf{D} \mathbf{J}_{\overline{\boldsymbol{\eta}}_{\mathrm{NLOS}}} \mathbf{D}^{\mathrm{T}}=\left[\begin{array}{ccc}
\mathbf{T}_{\mathbf{s}_{1}} \mathbf{J}_{\overline{\boldsymbol{\eta}}_{1}} \mathbf{T}_{\mathbf{s}_{1}}^{\mathrm{T}} \ldots & \mathbf{0} \\
\vdots & \ddots & \vdots \\
\mathbf{0} & \ldots \mathbf{T}_{\mathbf{s}_{K-1}} \mathbf{J}_{\overline{\boldsymbol{\eta}}_{K-1}} \mathbf{T}_{\mathbf{s}_{K-1}}^{\mathrm{T}}
\end{array}\right]
$$

For the compactness of notation, we use the following shorthand $\tilde{\mathbf{T}}_{\mathbf{s}_{k} \mathbf{s}_{k}} \triangleq \mathbf{T}_{\mathbf{s}_{k}} \mathbf{J}_{\overline{\boldsymbol{\eta}}_{k}} \mathbf{T}_{\mathbf{s}_{k}}^{\mathrm{T}}$ The inverse of a block diagonal matrix is determined by inverting the blocks on the diagonal. The $k^{\text {th }}$ block is given by

$$
\tilde{\mathbf{T}}_{\mathbf{s}_{k} \mathbf{s}_{k}} \triangleq\left[\begin{array}{cc}
a_{k} & b_{k} \\
b_{k} & d_{k}
\end{array}\right]
$$

where

$$
\begin{aligned}
a_{k} \triangleq & \frac{\left(\cos \left(\theta_{\mathrm{TX}, k}\right)+\cos \left(\theta_{\mathrm{RX}, k}\right)\right)^{2}}{\sigma_{\tau_{k}}^{2} c^{2}}+\frac{\sin ^{2}\left(\theta_{\mathrm{TX}, k}\right)}{\sigma_{\theta_{\mathrm{TX}, k}}^{2}\left\|\mathbf{q}-\mathbf{s}_{\mathbf{k}}\right\|^{2}}+ \\
& \frac{\sin ^{2}\left(\theta_{\mathrm{RX}, k}\right)}{\sigma_{\theta_{\mathrm{RX}, k}}^{2}\left\|\mathbf{p}-\mathbf{s}_{\mathbf{k}}\right\|^{2}} \\
b_{k} \triangleq & \frac{\left(\cos \left(\theta_{\mathrm{TX}, k}\right)+\cos \left(\theta_{\mathrm{RX}, k}\right)\right)\left(\sin \left(\theta_{\mathrm{TX}, k}\right)+\sin \left(\theta_{\mathrm{RX}, k}\right)\right)}{\sigma_{\tau_{k}}^{2} c^{2}} \\
& -\frac{\sin \left(\theta_{\mathrm{TX}, k}\right) \cos \left(\theta_{\mathrm{TX}, k}\right)}{\sigma_{\theta_{\mathrm{TX}, k}}^{2}\left\|\mathbf{q}-\mathbf{s}_{\mathbf{k}}\right\|^{2}}-\frac{\sin \left(\theta_{\mathrm{RX}, k}\right) \cos \left(\theta_{\mathrm{RX}, k}\right)}{\sigma_{\theta_{\mathrm{RX}, k}}^{2}\left\|\mathbf{p}-\mathbf{s}_{\mathbf{k}}\right\|^{2}}
\end{aligned}
$$




$$
\begin{gathered}
w_{R, k}^{(\mathrm{L})} \triangleq \frac{1}{a_{k} d_{k}-b_{k}^{2}}\left(\frac{1+\cos \left(\Delta \theta_{k}\right)}{\sigma_{\tau_{k}}^{2} c^{2}}\right)^{2}\left(\frac{1}{\sigma_{\theta_{\mathrm{RX}, k}}^{2}\left\|\mathbf{p}-\mathbf{s}_{\mathbf{k}}\right\|^{2}}+\frac{1}{\sigma_{\theta_{\mathrm{TX}, k}}^{2}\left\|\mathbf{q}-\mathbf{s}_{\mathbf{k}}\right\|^{2}}\right) \\
w_{A, k}^{(\mathrm{L})} \triangleq\left(\frac{\left(1+\cos \left(\Delta \theta_{k}\right)\right)^{2}}{\sigma_{\tau_{k}}^{2} c^{2} \sigma_{\theta_{\mathrm{RX}, k}^{4}}^{4}\left\|\mathbf{p}-\mathbf{s}_{\mathbf{k}}\right\|^{4}}+\frac{\sin ^{2}\left(\Delta \theta_{k}\right)}{\sigma_{\theta_{\mathrm{TX}, k}}^{2}\left\|\mathbf{q}-\mathbf{s}_{\mathbf{k}}\right\|^{2} \sigma_{\theta_{\mathrm{RX}, k}^{4}}^{4}\left\|\mathbf{p}-\mathbf{s}_{\mathbf{k}}\right\|^{4}}\right) \frac{1}{a_{k} d_{k}-b_{k}^{2}} \\
\gamma_{\mathbf{s}_{k}} \triangleq \frac{\left(1+\cos \left(\Delta \theta_{k}\right)\right) \sin \left(\Delta \theta_{k}\right)}{\sigma_{\theta_{\mathrm{TX}, k}}^{2}\left\|\mathbf{q}-\mathbf{s}_{\mathbf{k}}\right\|^{2} \sigma_{\theta_{\mathrm{RX}, k}}^{2}\left\|\mathbf{p}-\mathbf{s}_{\mathbf{k}}\right\|^{2} \sigma_{\tau_{k}}^{2} c^{2}} \frac{1}{a_{k} d_{k}-b_{k}^{2}} \\
\mathbf{B}_{k}^{(\mathrm{L}) \triangleq}\left[\begin{array}{ccc}
-2 \sin \left(\theta_{\mathrm{RX}, k}\right) \cos \left(\theta_{\mathrm{RX}, k}\right) & \cos ^{2}\left(\theta_{\mathrm{RX}, k}\right)-\sin ^{2}\left(\theta_{\mathrm{RX}, k}\right) & \cos \left(\theta_{\mathrm{RX}, k}\right)\left\|\mathbf{p}-\mathbf{s}_{k}\right\| \\
\cos ^{2}\left(\theta_{\mathrm{RX}, k}\right)-\sin ^{2}\left(\theta_{\mathrm{RX}, k}\right) & 2 \sin \left(\theta_{\mathrm{RX}, k}\right) \cos \left(\theta_{\mathrm{RX}, k}\right) & \sin \left(\theta_{\mathrm{RX}, k}\right)\left\|\mathbf{p}-\mathbf{s}_{k}\right\| \\
\cos \left(\theta_{\mathrm{RX}, k}\right)\left\|\mathbf{p}-\mathbf{s}_{k}\right\| & \sin \left(\theta_{\mathrm{RX}, k}\right)\left\|\mathbf{p}-\mathbf{s}_{k}\right\| & 0
\end{array}\right]
\end{gathered}
$$

$$
\begin{aligned}
d_{k} \triangleq & \frac{\left(\sin \left(\theta_{\mathrm{TX}, k}\right)+\sin \left(\theta_{\mathrm{RX}, k}\right)\right)^{2}}{\sigma_{\tau_{k}}^{2} c^{2}}+\frac{\cos ^{2}\left(\theta_{\mathrm{TX}, k}\right)}{\sigma_{\theta_{\mathrm{TX}, k}}^{2}\left\|\mathbf{q}-\mathbf{s}_{\mathbf{k}}\right\|^{2}}+ \\
& \frac{\cos ^{2}\left(\theta_{\mathrm{RX}, k}\right)}{\sigma_{\theta_{\mathrm{RX}, k}}^{2}\left\|\mathbf{p}-\mathbf{s}_{\mathbf{k}}\right\|^{2}} .
\end{aligned}
$$

Thus, the inverse of the $k^{\text {th }}$ block is given by

$$
\tilde{\mathbf{T}}_{\mathbf{s}_{k} \mathbf{s}_{k}}^{-1}=\frac{1}{\left|\tilde{\mathbf{T}}_{\mathbf{s}_{k} \mathbf{s}_{k} \mid}\right|}\left[\begin{array}{cc}
d_{k} & -b_{k} \\
-b_{k} & a_{k}
\end{array}\right]
$$

where $\left|\tilde{\mathbf{T}}_{\mathbf{s}_{k} \mathbf{s}_{k}}\right|=a_{k} d_{k}-b_{k}^{2}$.

The left term $\mathbf{B} \mathbf{J}_{\overline{\boldsymbol{\eta}}_{\mathrm{NLOS}}} \mathbf{D}^{\mathrm{T}}$ and the right term $\mathbf{D} \mathbf{J}_{\overline{\boldsymbol{\eta}}_{\mathrm{NLOS}}} \mathbf{B}^{\mathrm{T}}$ in (29) can be evaluated by straightforward matrix-matrix multiplications. Observe that the left term can be obtained by taking the transpose of the right term. Due to the block diagonal structure of $\mathbf{D}$, we find

$$
\mathbf{D} \mathbf{J}_{\overline{\boldsymbol{\eta}}_{\mathrm{NLOS}}} \mathbf{B}^{\mathrm{T}}=\left[\begin{array}{c}
\mathbf{T}_{\mathbf{s}_{1}} \mathbf{J}_{\overline{\boldsymbol{\eta}}_{1}}\left(\mathbf{T}_{\mathbf{P}}^{(1)}\right)^{\mathrm{T}} \\
\vdots \\
\mathbf{T}_{\mathbf{s}_{K-1}} \mathbf{J}_{\overline{\boldsymbol{\eta}}_{K-1}}\left(\mathbf{T}_{\mathbf{P}}^{(K-1)}\right)^{\mathrm{T}}
\end{array}\right] .
$$

We introduce a similar shorthand as above, where $\tilde{\mathbf{T}}_{\mathbf{s}_{k} \mathbf{P}} \triangleq$ $\mathbf{T}_{\mathbf{s}_{k}} \mathbf{J}_{\overline{\boldsymbol{\eta}}_{k}}\left(\mathbf{T}_{\mathbf{P}}^{(k)}\right)^{\mathrm{T}}$. Considering (55) and (56), we deduce

$$
\tilde{\mathbf{B}}^{(\mathrm{L})}=\sum_{k=1}^{K-1} \tilde{\mathbf{T}}_{\mathbf{s}_{k} \mathbf{P}}^{\mathrm{T}} \tilde{\mathbf{T}}_{\mathbf{s}_{k} \mathbf{s}_{k}}^{-1} \tilde{\mathbf{T}}_{\mathbf{s}_{k} \mathbf{P}} .
$$

The weights used in (36) are defined in (53) at the top of this page. In addition, we define the matrix in (54) at the top of this page. Using straightforward algebra and matrix-matrix multiplications, (36) is readily obtained.

\section{Net NLOS Gain}

We collect all information associated with the $k^{\text {th }}$ NLOS component in the matrix $\boldsymbol{\Psi}_{\mathbf{s}_{k}}$, i.e.

$$
\begin{aligned}
\boldsymbol{\Psi}_{\mathbf{s}_{k}} \triangleq & \epsilon_{\mathbf{s}_{k}} \boldsymbol{\Upsilon}_{0,0}\left(\theta_{\mathrm{TX}, 0}, 0,0\right) \\
& +\beta_{\mathbf{s}_{k}} \boldsymbol{\Upsilon}_{1,1}\left(\theta_{\mathrm{RX}, k}, \pi / 2,\|\mathbf{p}-\mathbf{q}\|\right)+\gamma_{\mathbf{s}_{k}} \mathbf{B}_{k}^{(\mathrm{L})} \\
\triangleq & {\left[\boldsymbol{\psi}_{\mathbf{s}_{k}, 1}, \boldsymbol{\psi}_{\mathbf{s}_{k}, 2}, \boldsymbol{\psi}_{\mathbf{s}_{k}, 3}\right] }
\end{aligned}
$$

We will now show that $\boldsymbol{\Psi}_{\mathbf{s}_{k}}$ has only one non-zero eigenvalue by showing that $\boldsymbol{\Psi}_{\mathbf{s}_{k}}$ is rank one, i.e. $\boldsymbol{\Psi}_{\mathbf{s}_{k}}$ has only one linearly independent column. By inspection of the columns of $\boldsymbol{\Psi}_{\mathbf{s}_{k}}$, it is straightforward to show that

$$
a \boldsymbol{\psi}_{\mathbf{s}_{k}, 1}-b \boldsymbol{\psi}_{\mathbf{s}_{k}, 2}=\left[\begin{array}{c}
\cos \left(\theta_{\mathrm{RX}, k}\right)\left\|\mathbf{p}-\mathbf{s}_{k}\right\|\left(\epsilon_{\mathbf{s}_{k}} \beta_{\mathbf{s}_{k}}-\gamma_{\mathbf{s}_{k}}^{2}\right) \\
\sin \left(\theta_{\mathrm{RX}, k}\right)\left\|\mathbf{p}-\mathbf{s}_{k}\right\|\left(\epsilon_{\mathbf{s}_{k}} \beta_{\mathbf{s}_{k}}-\gamma_{\mathbf{s}_{k}}^{2}\right) \\
0
\end{array}\right]
$$

where $a \triangleq\left[\Psi_{\mathbf{s}_{k}}\right]_{2,3}=\left(\beta_{\mathbf{s}_{k}} \cos \left(\theta_{\mathrm{RX}, k}\right)\left\|\mathbf{p}-\mathbf{s}_{k}\right\|+\right.$ $\left.\gamma_{\mathbf{s}_{k}} \sin \left(\theta_{\mathrm{RX}, k}\right)\left\|\mathbf{p}-\mathbf{s}_{k}\right\|\right)$ and $b \triangleq\left[\boldsymbol{\Psi}_{\mathbf{s}_{k}}\right]_{1,3}=$ $\left(-\beta_{\mathbf{s}_{k}} \sin \left(\theta_{\mathrm{RX}, k}\right)\left\|\mathbf{p}-\mathbf{s}_{k}\right\|+\gamma_{\mathbf{s}_{k}} \cos \left(\theta_{\mathrm{RX}, k}\right)\left\|\mathbf{p}-\mathbf{s}_{k}\right\|\right)$. Using simple algebra it can be seen that

$$
\left(\epsilon_{\mathbf{s}_{k}} \beta_{\mathbf{s}_{k}}-\gamma_{\mathbf{s}_{k}}^{2}\right)=0 .
$$

Hence $\boldsymbol{\psi}_{\mathbf{s}_{k}, 1}$ and $\boldsymbol{\psi}_{\mathbf{s}_{k}, 2}$ are linearly dependent. All other combinations of the columns of $\boldsymbol{\Psi}_{\mathbf{s}_{k}}$ can be shown to linearly dependent in the same fashion. Hence $\boldsymbol{\Psi}_{\mathbf{s}_{k}}$ is rank one and has one non-zero eigenvalue. The only non-zero eigenvalue is given by the trace of $\boldsymbol{\Psi}_{\mathbf{s}_{k}}$ [44]

$$
\lambda_{\mathbf{s}_{k}}=\epsilon_{\mathbf{s}_{k}}+\beta_{\mathbf{s}_{k}}\left(1+\left\|\mathbf{p}-\mathbf{s}_{k}\right\|\right) .
$$

Note that the terms $\epsilon_{\mathbf{s}_{k}}$ and $\beta_{\mathbf{s}_{k}}$ in (61) are functions of $\theta_{\mathrm{RX}, k}$, $\theta_{\mathrm{TX}, k},\left\|\mathbf{q}-\mathbf{s}_{k}\right\|,\left\|\mathbf{s}_{k}-\mathbf{p}\right\|, \sigma_{\mathrm{RX}, k}, \sigma_{\mathrm{TX}, k}$, and $\sigma_{\tau_{k}}$, i.e. the eigenvalue in (61) is a function of the geometry of the scenario and the qualities of the observations (AOA, AOD, and TOA). This results is presented in a more obvious format in (37).

Using the results for $\epsilon_{\mathbf{s}_{k}}$, and $\beta_{\mathbf{s}_{k}}$, as well as straightforward algebraic manipulations, the main result of this paper in (37) is readily obtained. That being said, it can be seen that

$$
\mathbf{v}_{\mathbf{s}_{k}}=\left[\begin{array}{c}
-\frac{\epsilon_{\mathbf{s}_{k}}}{\gamma_{\mathbf{s}_{k}}} \cos \left(\theta_{\mathrm{RX}, k}\right)-\sin \left(\theta_{\mathrm{RX}, k}\right) \\
-\frac{\epsilon_{\mathbf{s}_{k}}}{\gamma_{\mathbf{s}_{k}}} \sin \left(\theta_{\mathrm{RX}, k}\right)+\cos \left(\theta_{\mathrm{RX}, k}\right) \\
\left\|\mathbf{p}-\mathbf{s}_{k}\right\|
\end{array}\right] .
$$

is in the null space of $\left(\epsilon_{\mathbf{s}_{k}}+\beta_{\mathbf{s}_{k}}\left(1+\left\|\mathbf{p}-\mathbf{s}_{k}\right\|\right)\right) \mathbf{I}-\mathbf{\Psi}_{\mathbf{s}_{k}}$, i.e.

$$
\left(\left(\epsilon_{\mathbf{s}_{k}}+\beta_{\mathbf{s}_{k}}\left(1+\left\|\mathbf{p}-\mathbf{s}_{k}\right\|\right)\right) \mathbf{I}-\mathbf{\Psi}_{\mathbf{s}_{k}}\right) \mathbf{v}_{\mathbf{s}_{k}}=\mathbf{0} .
$$

Hence $\mathbf{v}_{\mathbf{s}_{k}}$ is the eigenvector corresponding to the eigenvalue in (61). 


\section{REFERENCES}

[1] Y. Han, Y. Shen, X. P. Zhang, M. Z. Win, and H. Meng, "Performance limits and geometric properties of array localization," IEEE Transactions on Information Theory, vol. 62, no. 2, pp. 1054-1075, Feb 2016.

[2] Y. Shen and M. Z. Win, "Fundamental limits of wideband localization; part I: A general framework," IEEE Transactions on Information Theory, vol. 56, no. 10, pp. 4956-4980, Oct 2010.

[3] Y. Shen, H. Wymeersch, and M. Z. Win, "Fundamental limits of wideband localization; part II: Cooperative networks," IEEE Transactions on Information Theory, vol. 56, no. 10, pp. 4981-5000, Oct 2010.

[4] Y. Shen and M. Z. Win, "On the accuracy of localization systems using wideband antenna arrays," IEEE Transactions on Communications, vol. 58, no. 1, pp. 270-280, January 2010.

[5] M. Z. Win, Y. Shen, and W. Dai, "A theoretical foundation of network localization and navigation," Proceedings of the IEEE, vol. 106, no. 7, pp. 1136-1165, Jul. 2018, special issue on Foundations and Trends in Localization Technologies.

[6] J. G. Andrews, S. Buzzi, W. Choi, S. V. Hanly, A. Lozano, A. C. K. Soong, and J. C. Zhang, "What will 5G be?" IEEE Journal on Selected Areas in Communications, vol. 32, no. 6, pp. 1065-1082, June 2014.

[7] S. Rangan, T. S. Rappaport, and E. Erkip, "Millimeter-wave cellular wireless networks: Potentials and challenges," Proceedings of the IEEE, vol. 102, no. 3, pp. 366-385, March 2014.

[8] A. Ghosh, T. A. Thomas, M. C. Cudak, R. Ratasuk, P. Moorut, F. W. Vook, T. S. Rappaport, G. R. MacCartney, S. Sun, and S. Nie, "Millimeter-wave enhanced local area systems: A high-data-rate approach for future wireless networks," IEEE Journal on Selected Areas in Communications, vol. 32, no. 6, pp. 1152-1163, June 2014.

[9] Z. Pi and F. Khan, "An introduction to millimeter-wave mobile broadband systems," IEEE Communications Magazine, vol. 49, no. 6, pp. 101-107, June 2011.

[10] T. S. Rappaport, S. Sun, R. Mayzus, H. Zhao, Y. Azar, K. Wang, G. N. Wong, J. K. Schulz, M. Samimi, and F. Gutierrez, "Millimeter wave mobile communications for 5G cellular: It will work!" IEEE Access, vol. 1, pp. 335-349, 2013.

[11] R. W. Heath, N. González-Prelcic, S. Rangan, W. Roh, and A. M. Sayeed, "An overview of signal processing techniques for millimeter wave MIMO systems," IEEE Journal of Selected Topics in Signal Processing, vol. 10, no. 3, pp. 436-453, April 2016.

[12] O. Orhan, E. Erkip, and S. Rangan, "Low power analog-to-digital conversion in millimeter wave systems: Impact of resolution and bandwidth on performance," in 2015 Information Theory and Applications Workshop (ITA), Feb 2015, pp. 191-198.

[13] T. Bai and R. W. Heath, "Coverage and rate analysis for millimeter-wave cellular networks," IEEE Transactions on Wireless Communications, vol. 14, no. 2, pp. 1100-1114, Feb 2015.

[14] N. Patwari, J. N. Ash, S. Kyperountas, A. O. Hero, R. L. Moses, and N. S. Correal, "Locating the nodes: cooperative localization in wireless sensor networks," IEEE Signal Processing Magazine, vol. 22, no. 4, pp. 54-69, July 2005.

[15] A. Shahmansoori, G. E. Garcia, G. Destino, G. Seco-Granados, and $\mathrm{H}$. Wymeersch, "5G position and orientation estimation through millimeter wave MIMO," in 2015 IEEE Globecom Workshops (GC Wkshps), Dec 2015, pp. 1-6.

[16] J. Li, J. Conan, and S. Pierre, "Joint estimation of channel parameters for MIMO communication systems," in 2005 2nd International Symposium on Wireless Communication Systems, Sept 2005, pp. 22-26.

[17] M. D. Larsen, A. L. Swindlehurst, and T. Svantesson, "Performance bounds for MIMO-OFDM channel estimation," IEEE Transactions on Signal Processing, vol. 57, no. 5, pp. 1901-1916, May 2009.

[18] A. Guerra, F. Guidi, and D. Dardari, "Single-anchor localization and orientation performance limits using massive arrays: MIMO vs.beamforming," IEEE Transactions on Wireless Communications, vol. 17, no. 8, pp. 5241-5255, Aug 2018.

[19] A. Shahmansoori, G. E. Garcia, G. Destino, G. Seco-Granados, and $\mathrm{H}$. Wymeersch, "Position and orientation estimation through millimeterwave MIMO in 5G systems," IEEE Transactions on Wireless Communications, vol. 17, no. 3, pp. 1822-1835, March 2018.

[20] Z. Abu-Shaban, X. Zhou, T. Abhayapala, G. Seco-Granados, and H. Wymeersch, "Error bounds for uplink and downlink 3D localization in 5G millimeter wave systems," IEEE Transactions on Wireless Communications, vol. 17, no. 8, pp. 4939-4954, Aug 2018.

[21] C. Gentner, T. Jost, W. Wang, S. Zhang, A. Dammann, and U. C. Fiebig, "Multipath assisted positioning with simultaneous localization and mapping," IEEE Transactions on Wireless Communications, vol. 15, no. 9, pp. 6104-6117, Sept 2016.
[22] C. Gentner, B. Ma, M. Ulmschneider, T. Jost, and A. Dammann, "Simultaneous localization and mapping in multipath environments," in 2016 IEEE/ION Position, Location and Navigation Symposium (PLANS), April 2016, pp. 807-815.

[23] E. Leitinger, F. Meyer, P. Meissner, K. Witrisal, and F. Hlawatsch, "Belief propagation based joint probabilistic data association for multipathassisted indoor navigation and tracking," in 2016 International Conference on Localization and GNSS (ICL-GNSS), June 2016, pp. 1-6.

[24] J. Kulmer, E. Leitinger, P. Meissner, S. Hinteregger, and K. Witrisal, "Cooperative localization and tracking using multipath channel information," in 2016 International Conference on Localization and GNSS (ICL-GNSS), June 2016, pp. 1-6.

[25] K. Witrisal, P. Meissner, E. Leitinger, Y. Shen, C. Gustafson, F. Tufvesson, K. Haneda, D. Dardari, A. F. Molisch, A. Conti, and M. Z Win, "High-accuracy localization for assisted living: 5G systems will turn multipath channels from foe to friend," IEEE Signal Processing Magazine, vol. 33, no. 2, pp. 59-70, March 2016.

[26] F. Meyer, T. Kropfreiter, J. L. Williams, R. A. Lau, F. Hlawatsch, P. Braca, and M. Z. Win, "Message passing algorithms for scalable multitarget tracking," Proceedings of the IEEE, vol. 106, no. 2, pp. 221259, Feb. 2018.

[27] Y. Shen and M. Z. Win, "Fundamental limits of wideband localization accuracy via Fisher information," in 2007 IEEE Wireless Communications and Networking Conference, March 2007, pp. 3046-3051.

[28] J.-Y. Lee and R. A. Scholtz, "Ranging in a dense multipath environment using an uwb radio link," IEEE Journal on Selected Areas in Coтmиnications, vol. 20, no. 9, pp. 1677-1683, Dec 2002.

[29] B. Zhen, H. B. Li, and R. Kohno, "Clock management in ultra-wideband ranging," in 2007 16th IST Mobile and Wireless Communications Summit, July 2007, pp. 1-5.

[30] H. V. Trees, Optimum Array Processing: Part IV of Detection, Estimation, and Modulation Theory. Wiley, 2002.

[31] T. S. Rappaport, G. R. MacCartney, M. K. Samimi, and S. Sun, "Wideband millimeter-wave propagation measurements and channel models for future wireless communication system design," IEEE Transactions on Communications, vol. 63, no. 9, pp. 3029-3056, Sept 2015.

[32] H. Deng and A. Sayeed, "Mm-wave MIMO channel modeling and user localization using sparse beamspace signatures," in 2014 IEEE 15th International Workshop on Signal Processing Advances in Wireless Communications (SPAWC), June 2014, pp. 130-134.

[33] O. Besson and P. Stoica, "Decoupled estimation of DOA and angular spread for a spatially distributed source," IEEE Transactions on Signal Processing, vol. 48, no. 7, pp. 1872-1882, July 2000.

[34] M. Fakharzadeh, M. R. Nezhad-Ahmadi, B. Biglarbegian, J. AhmadiShokouh, and S. Safavi-Naeini, "CMOS phased array transceiver technology for $60 \mathrm{GHz}$ wireless applications," IEEE Transactions on Antennas and Propagation, vol. 58, no. 4, pp. 1093-1104, April 2010.

[35] V. Venkateswaran and A. J. van der Veen, "Analog beamforming in MIMO communications with phase shift networks and online channel estimation," IEEE Transactions on Signal Processing, vol. 58, no. 8, pp. 4131-4143, Aug 2010.

[36] M. Giordani, M. Mezzavilla, and M. Zorzi, "Initial access in 5g mmwave cellular networks," IEEE Communications Magazine, vol. 54, no. 11, pp. 40-47, November 2016.

[37] Kay, Fundamentals of Statistical Signal Processing: Estimation Theory. NJ, USA: Prentice-Hall, Inc.,, 1993.

[38] J. S. Abel, "A bound on mean-square-estimate error," IEEE Transactions on Information Theory, vol. 39, no. 5, pp. 1675-1680, Sep 1993.

[39] H. V. Trees and K. L. Bell, Bayesian Bounds for Parameter Estimation and Nonlinear Filtering/Tracking. Wiley, 2007.

[40] H. V. Trees, K. L. Bell, and Z. Tian, Detection Estimation and Modulation Theory, Part I: Detection, Estimation, and Filtering Theory, 2nd Edition. Wiley, 2013.

[41] P. Tichavsky, C. H. Muravchik, and A. Nehorai, "Posterior CramerRao bounds for discrete-time nonlinear filtering," IEEE Transactions on Signal Processing, vol. 46, no. 5, pp. 1386-1396, May 1998.

[42] R. M. Buehrer, H. Wymeersch, and R. M. Vaghefi, "Collaborative sensor network localization: Algorithms and practical issues," Proceedings of the IEEE, vol. 106, no. 6, pp. 1089-1114, June 2018.

[43] A. Goldsmith, Wireless Communications. Cambridge University Press, 2005.

[44] A. J. Laub, Matrix Analysis for Scientists and Engineers. Society for Industrial and Applied Mathematics, 2004. 


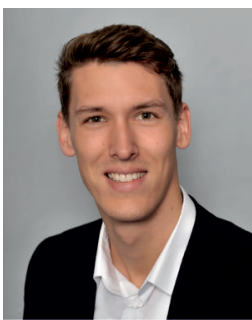

Rico Mendrzik (S'15) received a B.Sc. degree in 2012 and a M.Sc. degree in 2015 both in electrical engineering from Hamburg University of Technology (TUHH), Germany. He is currently working towards his Ph.D. degree at the Institute of Communications, Hamburg University of Technology, Germany. In 2017, he was a visiting researcher at the University of California, Los Angeles (UCLA) and he is currently affiliated with the Laboratory for Information and Decision Systems (LIDS) at the Massachusetts Institute of Technology (MIT) as visiting researcher. His main research interests are in the area of wireless positioning, Bayesian inference, estimation theory, and signal processing.

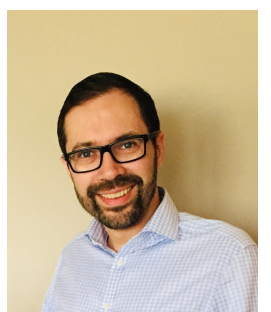

Henk Wymeersch (S'01, M'05) obtained the Ph.D. degree in Electrical Engineering/Applied Sciences in 2005 from Ghent University, Belgium. He is currently a Professor of Communication Systems with the Department of Electrical Engineering at Chalmers University of Technology, Sweden. Prior to joining Chalmers, he was a postdoctoral researcher from 2005 until 2009 with the Laboratory for Information and Decision Systems at the Massachusetts Institute of Technology. Prof. Wymeersch served as Associate Editor for IEEE Communication Letters (2009-2013), IEEE Transactions on Wireless Communications (since 2013), and IEEE Transactions on Communications (2017-2018). His current research interests include cooperative systems and intelligent transportation systems.

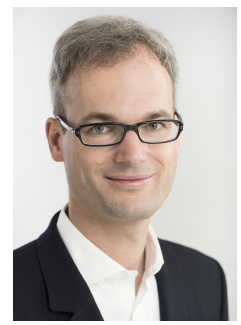

Gerhard Bauch received the Dipl.-Ing. and Dr.Ing. degree in Electrical Engineering from Munich University of Technology (TUM) in 1995 and 2001, respectively, and the Diplom-Volkswirt (master in economics) degree from FernUniversitaet Hagen in 2001. In 1996, he was with the German Aerospace Center (DLR), Oberpfaffenhofen, Germany. From 1996-2001 he was member of scientific staff at Munich University of Technology (TUM). In 1998 and 1999 he was also visiting researcher at AT\&T Labs Research, Florham Park, NJ, USA. In 2002 he joined DOCOMO Euro-Labs, Munich, Germany, where he has been managing the Advanced Radio Transmission Group. In 2007 he was additionally appointed Research Fellow of DOCOMO Euro-Labs. He was a full professor at the Universität der Bundeswehr Munich from 2009-2012. Since October 2012 he is head of the Institute of Communications at Hamburg University of Technology.

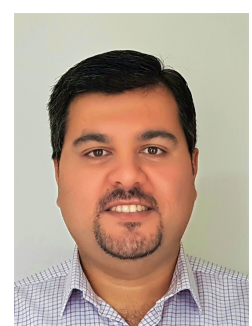

Zohair Abu-Shaban (S'14-M'17) is a Postdoctoral Research Fellow at the University of New South Wales, Australia. He obtained a $\mathrm{PhD}$ degree from the Australian National University (ANU) in 2018, and MSc from Imperial College London in 2010, both in electrical and electronic engineering. During his $\mathrm{PhD}$ he was a Visiting Research Scholar at the Universitat Autónoma de Barcelona, Spain, and Chalmers University of Technology, Sweden. Prior to starting his PhD, he was an Early-Stage Researcher at the University of Luxembourg, from January 2012 to May 2014, pursuing research in collaboration with SES S.A, funded by the National Research Fund, Luxembourg (FNR). During his career, he received several scholarships and awards including the Department Prize for Outstanding Achievement from Imperial College London, and the Marie Sklodowska-Curie Action (MSCA) research fellowship from the European Commission. He is also on the Executive Committee of the IEEE ACT Section as Chair for Young Professionals. 